\title{
Parton distributions from nonlocal chiral SU(3) effective theory: Splitting functions
}

\author{
Y. Salamu, ${ }^{1,2}$ Chueng-Ryong Ji, ${ }^{3}$ W. Melnitchouk, ${ }^{4}$ A. W. Thomas, ${ }^{5}$ and P. Wang ${ }^{1,6}$ \\ ${ }^{1}$ Institute of High Energy Physics, CAS, Beijing 100049, China \\ ${ }^{2}$ School of Physical Sciences, University of Chinese Academy of Sciences, Beijing 100049, China \\ ${ }^{3}$ North Carolina State University, Raleigh, North Carolina 27695, USA \\ ${ }^{4}$ Jefferson Lab, Newport News, Virginia 23606, USA \\ ${ }^{5}$ CoEPP and CSSM, Department of Physics, University of Adelaide, Adelaide SA 5005, Australia \\ ${ }^{6}$ Theoretical Physics Center for Science Facilities, CAS, Beijing 100049, China
}

(Received 20 June 2018; published 29 January 2019; corrected 24 July 2019)

\begin{abstract}
We present a new formulation of pseudoscalar meson loop corrections to nucleon parton distributions within a nonlocal covariant chiral effective field theory, including contributions from SU(3) octet and decuplet baryons. The nonlocal Lagrangian, constrained by requirements of local gauge invariance and Lorentz-invariant ultraviolet regularization, generates additional interactions associated with gauge links. We use these to compute the full set of proton $\rightarrow$ meson + baryon splitting functions, which in general contain on-shell and off-shell contributions, in addition to $\delta$-function terms at zero momentum, along with nonlocal contributions associated with the finite size of the proton. We illustrate the shapes of the various local and nonlocal functions numerically using a simple example of a dipole regulator.
\end{abstract}

DOI: 10.1103/PhysRevD.99.014041

\section{INTRODUCTION}

The important role played by chiral symmetry in hadron physics has been documented for many decades. Traditionally the purview of low-energy hadron and nuclear physics, more recently the relevance of chiral symmetry in QCD has become more prominent also in high-energy reactions, in which the quark and gluon (or parton) substructure of hadrons is manifest. One of the most striking expressions of the chiral symmetry and its approximate breaking is in the nonperturbative structure of the sea quark distributions of the nucleon [1,2]. In particular, the breaking of chiral SU(3) symmetry was anticipated [3] to generate unequal strange and (light) nonstrange sea quark distributions, and, even more dramatically, an excess of $\bar{d}$ antiquarks over $\bar{u}$. The latter was confirmed in proton-proton and proton-deuteron Drell-Yan experiments at CERN [4] and Fermilab [5], following earlier indirect indications from inclusive [6] and semi-inclusive [7] deep-inelastic scattering (DIS) data on proton and deuteron targets.

The observation of a large $\bar{d}-\bar{u}$ asymmetry has also served to motivate more challenging searches for other nonperturbative asymmetries, such as those between strange and antistrange quarks in the proton, $s-\bar{s}[8-10]$, or

Published by the American Physical Society under the terms of the Creative Commons Attribution 4.0 International license. Further distribution of this work must maintain attribution to the author(s) and the published article's title, journal citation, and DOI. Funded by SCOAP. between the helicity dependent light antiquark distributions, $\Delta \bar{d}-\Delta \bar{u}[11]$. The phenomenological success in describing the $\bar{d}-\bar{u}$ asymmetry, in particular, in terms of nonperturbative models of the nucleon in which its peripheral structure is modeled by a pseudoscalar meson cloud suggested that signatures of chiral symmetry breaking may also be found in other types of parton distribution functions (PDFs) [8,12-18].

While considerable experience has been accumulated with nonperturbative models, a challenge has been to compute the chiral symmetry breaking effects on the PDFs in a model-independent way from QCD. An important step in establishing a direct connection with QCD was made with the observation [19] that the leading nonanalytic (LNA) behavior of moments of the nonsinglet PDFs, expanded in powers of the pion mass, $m_{\pi}$, could be obtained from chiral effective field theory, which encodes the same chiral symmetry properties as present in QCD [20-22]. In addition to demonstrating how lattice QCD data on PDF moments and other observables simulated at unphysically large pion masses could be extrapolated to the physical point [23], the result [19] demonstrated unambiguously that a nonzero component of $\bar{d}-\bar{u}$ arises as a direct consequence of the infrared structure of QCD.

Subsequent work [24-29] computed the full set of lowest order corrections to PDFs arising from pseudoscalar meson loops, both for the PDF moments and the Bjorken- $x$ dependence. The LNA behavior of the various contributions can be established model-independently by considering the infrared limit; however, the computation of the 
full amplitude requires specific choices for regularizing the divergences in the loop integrals. In the literature, regularization prescriptions such as transverse momentum cutoffs, Pauli-Villars, dimensional regularization or infrared regularization have been used, as well as form factors or finite-range regulators. The latter take into account the finite size of hadrons [30,31], while the others are generally more suitable for theories that treat hadrons as pointlike.

In practice, the extended structure of the nucleon and other baryons does become important in many traditional hadronic physics applications. In nonrelativistic calculations, if the regulators are in three-dimensional momentum space, such as for finite-range regularization, charge conservation, which is related to the time component of the current, is respected in the presence of form factors. In relativistic calculations, on the other hand, simply replacing the nonrelativistic regulator by a covariant one can lead to violation of local gauge symmetry and charge conservation.

The problem of preserving gauge invariance in theories with hadronic form factors can be formally alleviated by introducing nonlocal interactions into the gauge invariant local Lagrangian, which allows one to consistently generate a covariant regulator. A method for constructing nonlocal Lagrangians with gauge fields was described by Terning [32], based on the path-ordered exponential introduced by Wilson [33] and earlier by Bloch [34]. Variants of the method were subsequently used in phenomenological applications to strange vector form factors and other nucleon matrix elements by a number of authors [35-37]. The pion and $\sigma$ meson properties have been studied by gauging nonlocal meson-quark interactions in relativistic quark models [38,39]. The nonlocal Lagrangian at the hadron level was also recently constructed and applied to electromagnetic form factors of the nucleon [40-42].

The presence of gauge links in the nonlocal Lagrangian connecting different spacetime coordinates generates additional diagrams which are needed to ensure the local gauge invariance of the theory. This guarantees that the proton and neutron charges, for example, are unaffected by meson loops, or that contributions to the strangeness in the nucleon from diagrams with intermediate state kaons and hyperons sum to zero. These basic features of the theory are not guaranteed for a local Lagrangian with a covariant regulator, but arise automatically in the nonlocal theory in which the Ward identities and charge conservation are necessarily satisfied. In fact, a nonlocal formulation may be preferable on physical grounds, as this more naturally represents the extended structure of hadrons.

In this paper we describe how the nonlocal formulation of the chiral SU(3) effective theory can be used to derive the contributions from pseudoscalar meson loops to PDFs in the nucleon. We include both the SU(3) octet and decuplet baryons, using a covariant regulator generated through the nonlocal Lagrangian that respects Lorentz and gauge symmetry. In the present paper we focus on the formalism and the derivation of the proton $\rightarrow$ baryon + meson splitting functions from the nonlocal chiral Lagrangian; a follow-up paper [43] will report on the results for the nucleon PDFs, computed through convolutions of the splitting functions and PDFs in the virtual mesons and baryons in the loops.

We begin by reviewing in Sec. II the familiar local effective Lagrangian in the standard chiral SU(3) effective field theory. The generalization of the effective Lagrangian to the nonlocal case is described in Sec. III, a procedure which allows the preservation of gauge invariance in the presence of covariant vertex functions for the nucleonbaryon-meson interaction. The main results for the proton $\rightarrow$ meson + baryon splitting functions are derived in Sec. IV for the full set of lowest order diagrams, including rainbow, bubble, tadpole and Kroll-Ruderman contributions, as well as additional terms that arise from the gauge links generated from the nonlocal interactions. Here we present the model independent results for the nonanalytic behavior of the moments of the splitting functions, and illustrate the relative shapes and magnitudes of the various functions using a simple example of a covariant dipole vertex form factor. Finally, in Sec. V we summarize our results and outline future applications of the new formalism.

\section{LOCAL CHIRAL EFFECTIVE LAGRANGIAN}

In this section we review the standard local chiral effective theory for mesons and baryons. The lowestorder Lagrangian, consistent with chiral $\mathrm{SU}(3)_{L} \times$ $\mathrm{SU}(3)_{R}$ symmetry, describing the interaction of pseudoscalar mesons $(\phi)$ with octet $(B)$ and decuplet $\left(T_{\mu}\right)$ baryons, is given by $[44,45]$

$$
\begin{aligned}
\mathcal{L}= & \operatorname{Tr}\left[\bar{B}\left(i \not D-M_{B}\right) B\right]-\frac{D}{2} \operatorname{Tr}\left[\bar{B} \gamma^{\mu} \gamma_{5}\left\{u_{\mu}, B\right\}\right] \\
& -\frac{F}{2} \operatorname{Tr}\left[\bar{B} \gamma^{\mu} \gamma_{5}\left[u_{\mu}, B\right]\right]+\bar{T}_{\mu}^{i j k}\left(i \gamma^{\mu \nu \alpha} D_{\alpha}-M_{T} \gamma^{\mu \nu}\right) T_{\nu}^{i j k} \\
& -\frac{\mathcal{C}}{2}\left[\epsilon^{i j k} \bar{T}_{\mu}^{i l m} \Theta^{\mu \nu}\left(u_{\nu}\right)^{l j} B^{m k}+\text { H.c. }\right] \\
& -\frac{\mathcal{H}}{2} \bar{T}_{\mu}^{i j k} \gamma^{\alpha} \gamma_{5}\left(u_{\alpha}\right)^{k l} T_{\mu}^{i j l}+\frac{f^{2}}{4} \operatorname{Tr}\left[D_{\mu} U\left(D^{\mu} U\right)^{\dagger}\right],
\end{aligned}
$$

where $M_{B}$ and $M_{T}$ are the octet and decuplet masses, $D$ and $F$ are the meson-octet baryon coupling constants, $\mathcal{C}$ and $\mathcal{H}$ are the meson-octet-decuplet and meson-decupletdecuplet baryon couplings, respectively, $f=93 \mathrm{MeV}$ is the pseudoscalar decay constant, and "H.c." denotes the Hermitian conjugate. The tensor $\epsilon^{i j k}$ is the antisymmetric tensor in flavor space, and we define the tensors $\gamma^{\mu \nu}=$ $\frac{1}{2}\left[\gamma^{\mu}, \gamma^{\nu}\right]$ and $\gamma^{\mu \nu \alpha}=\frac{1}{2}\left\{\gamma^{\mu \nu}, \gamma^{\alpha}\right\}$ in terms of the Dirac $\gamma$ matrices. The octet-decuplet transition tensor operator $\Theta^{\mu \nu}$ is defined as 


$$
\Theta^{\mu \nu}=g^{\mu \nu}-\left(Z+\frac{1}{2}\right) \gamma^{\mu} \gamma^{\nu}
$$

where $Z$ is the decuplet off-shell parameter. The SU(3) baryon octet fields $B^{i j}$ include the nucleon $N(=p, n), \Lambda$, $\Sigma^{ \pm, 0}$ and $\Xi^{-, 0}$ fields, and are given by the matrix

$$
B=\left(\begin{array}{ccc}
\frac{1}{\sqrt{2}} \Sigma^{0}+\frac{1}{\sqrt{6}} \Lambda & \Sigma^{+} & p \\
\Sigma^{-} & -\frac{1}{\sqrt{2}} \Sigma^{0}+\frac{1}{\sqrt{6}} \Lambda & n \\
\Xi^{-} & \Xi^{0} & -\frac{2}{\sqrt{6}} \Lambda
\end{array}\right) .
$$

The baryon decuplet fields $T_{\mu}^{i j k}$, which include the $\Delta, \Sigma^{*}$, $\Xi^{*}$ and $\Omega^{-}$fields, are represented by symmetric tensors with components

$T^{111}=\Delta^{++}, \quad T^{112}=\frac{1}{\sqrt{3}} \Delta^{+}, \quad T^{122}=\frac{1}{\sqrt{3}} \Delta^{0}, \quad T^{222}=\Delta^{-}$, $T^{113}=\frac{1}{\sqrt{3}} \Sigma^{*+}, \quad T^{123}=\frac{1}{\sqrt{6}} \Sigma^{* 0}, \quad T^{223}=\frac{1}{\sqrt{3}} \Sigma^{*-}$,

$T^{133}=\frac{1}{\sqrt{3}} \Xi^{* 0}, \quad T^{233}=\frac{1}{\sqrt{3}} \Xi^{*-}$,

$T^{333}=\Omega^{-}$.

In the meson sector, the operator $U$ in Eq. (1) is defined in terms of the matrix of pseudoscalar fields $\phi$,

$$
U=u^{2}, \quad \text { with } \quad u=\exp \left(i \frac{\phi}{\sqrt{2} f}\right),
$$

where $\phi$ includes the $\pi, K$ and $\eta$ mesons,

$$
\phi=\left(\begin{array}{ccc}
\frac{1}{\sqrt{2}} \pi^{0}+\frac{1}{\sqrt{6}} \eta & \pi^{+} & K^{+} \\
\pi^{-} & -\frac{1}{\sqrt{2}} \pi^{0}+\frac{1}{\sqrt{6}} \eta & K^{0} \\
K^{-} & \bar{K}^{0} & -\frac{2}{\sqrt{6}} \eta
\end{array}\right) .
$$

The pseudoscalar mesons couple to the baryon fields through the vector and axial vector combinations

$$
\Gamma_{\mu}=\frac{1}{2}\left(u^{\dagger} \partial_{\mu} u+u \partial_{\mu} u^{\dagger}\right)-\frac{i}{2}\left(u^{\dagger} \lambda^{a} u+u \lambda^{a} u^{\dagger}\right) v_{\mu}^{a},
$$

$$
u_{\mu}=i\left(u^{\dagger} \partial_{\mu} u-u \partial_{\mu} u^{\dagger}\right)+\left(u^{\dagger} \lambda^{a} u-u \lambda^{a} u^{\dagger}\right) v_{\mu}^{a},
$$

where $v_{\mu}^{a}$ corresponds to an external vector field, and $\lambda^{a}$ $(a=1, \ldots, 8)$ are the Gell-Mann matrices. The covariant derivatives of the octet and decuplet baryon fields in the chiral Lagrangian (1) are defined as $[46,47]$

$$
\begin{gathered}
D_{\mu} B=\partial_{\mu} B+\left[\Gamma_{\mu}, B\right]-i\left\langle\lambda^{0}\right\rangle v_{\mu}^{0} B, \\
D_{\mu} T_{\nu}^{i j k}=\partial_{\mu} T_{\nu}^{i j k}+\left(\Gamma_{\mu}, T_{\nu}\right)^{i j k}-i\left\langle\lambda^{0}\right\rangle v_{\mu}^{0} T_{\nu}^{i j k},
\end{gathered}
$$

where $v_{\mu}^{0}$ denotes an external singlet vector field, $\lambda^{0}$ is the unit matrix, and $\langle\cdots\rangle$ denotes a trace in flavor space. For the covariant derivative of the decuplet field, we use the notation

$$
\left(\Gamma_{\mu}, T_{\nu}\right)^{i j k}=\left(\Gamma_{\mu}\right)_{l}^{i} T_{\nu}^{l j k}+\left(\Gamma_{\mu}\right)_{l}^{j} T_{\nu}^{i l k}+\left(\Gamma_{\mu}\right)_{l}^{k} T_{\nu}^{i j l} .
$$

For the pseudoscalar meson fields, the covariant derivarive is written

$$
D_{\mu} U=\partial_{\mu} U+\left(i U \lambda^{a}-i \lambda^{a} U\right) v_{\mu}^{a} .
$$

Expanding the Lagrangian (1) to leading order in the baryon and meson fields, the relevant interaction part for a meson and baryon coupling to a proton can be written explicitly as

$$
\begin{aligned}
\mathcal{L}_{\text {int }}= & \frac{(D+F)}{2 f}\left(\bar{p} \gamma^{\mu} \gamma^{5} p \partial_{\mu} \pi^{0}+\sqrt{2} \bar{p} \gamma^{\mu} \gamma^{5} n \partial_{\mu} \pi^{+}\right)-\frac{(D+3 F)}{\sqrt{12} f} \bar{p} \gamma^{\mu} \gamma^{5} \Lambda \partial_{\mu} K^{+} \\
& +\frac{(D-F)}{2 f}\left(\sqrt{2} \bar{p} \gamma^{\mu} \gamma^{5} \Sigma^{+} \partial_{\mu} K^{0}+\bar{p} \gamma^{\mu} \gamma^{5} \Sigma^{0} \partial_{\mu} K^{+}\right)-\frac{D-3 F}{\sqrt{12} f} \bar{p} \gamma^{\mu} \gamma^{5} p \partial_{\mu} \eta \\
& +\frac{\mathcal{C}}{\sqrt{12} f}\left(-2 \bar{p} \Theta^{\nu \mu} \Delta_{\mu}^{+} \partial_{\nu} \pi^{0}-\sqrt{2} \bar{p} \Theta^{\nu \mu} \Delta_{\mu}^{0} \partial_{\nu} \pi^{+}+\sqrt{6} \bar{p} \Theta^{\nu \mu} \Delta_{\mu}^{++} \partial_{\nu} \pi^{-}-\bar{p} \Theta^{\nu \mu} \Sigma_{\mu}^{* 0} \partial_{\nu} K^{+}+\sqrt{2} \bar{p} \Theta^{\nu \mu} \Sigma_{\mu}^{*+} \partial_{\nu} K^{0}+\text { H.c. }\right) \\
& +\frac{i}{4 f^{2}} \bar{p} \gamma^{\mu} p\left[\left(\pi^{+} \partial_{\mu} \pi^{-}-\pi^{-} \partial_{\mu} \pi^{+}\right)+2\left(K^{+} \partial_{\mu} K^{-}-K^{-} \partial_{\mu} K^{+}\right)+\left(K^{0} \partial_{\mu} \bar{K}^{0}-\bar{K}^{0} \partial_{\mu} K^{0}\right)\right] .
\end{aligned}
$$

The terms involving the coupling $\mathcal{H}$ are not present because of the restriction to proton initial states. The current calculations below also do not involve the terms with the coupling $\mathcal{H}$ for the proton initial states. 
From the Lagrangian (1) one can also obtain the form of the electromagnetic current that couples to the external field $v_{\mu}^{a}$,

$$
\begin{aligned}
J_{a}^{\mu}= & \frac{1}{2} \operatorname{Tr}\left[\bar{B} \gamma^{\mu}\left[u \lambda^{a} u^{\dagger}+u^{\dagger} \lambda^{a} u, B\right]+\frac{D}{2} \operatorname{Tr}\left[\bar{B} \gamma^{\mu} \gamma_{5}\left\{u \lambda^{a} u^{\dagger}-u^{\dagger} \lambda^{a} u, B\right\}\right]+\frac{F}{2} \operatorname{Tr}\left[\bar{B} \gamma^{\mu} \gamma_{5}\left[u \lambda^{a} u^{\dagger}-u^{\dagger} \lambda^{a} u, B\right]\right]\right. \\
& +\frac{1}{2} \bar{T}_{\nu} \gamma^{\nu \alpha \mu}\left(u \lambda^{a} u^{\dagger}+u^{\dagger} \lambda^{a} u, T_{\alpha}\right)+\frac{\mathcal{C}}{2}\left(\bar{T}_{\nu} \Theta^{\nu \mu}\left(u \lambda^{a} u^{\dagger}-u^{\dagger} \lambda^{a} u\right) B+\text { H.c. }\right) \\
& +\frac{f^{2}}{4} \operatorname{Tr}\left[\partial^{\mu} U\left(U^{\dagger} i \lambda^{a}-i \lambda^{a} U^{\dagger}\right)+\left(U i \lambda^{a}-i \lambda^{a} U\right) \partial^{\mu} U^{\dagger}\right] .
\end{aligned}
$$

For the SU(3) flavor singlet current coupling to the external field $v_{\mu}^{0}$, one has

$$
J_{0}^{\mu}=\left\langle\lambda^{0}\right\rangle \operatorname{Tr}\left[\bar{B} \gamma^{\mu} B\right]+\left\langle\lambda^{0}\right\rangle \bar{T}_{\nu} \gamma^{\nu \alpha \mu} T_{\alpha},
$$

where again $\lambda^{0}$ is the unit matrix and $\langle\cdots\rangle$ denotes a trace in flavor space.

The currents for a given quark flavor are then expressed as combinations of the SU(3) singlet and octet currents,

$$
\begin{aligned}
& J_{u}^{\mu}=\frac{1}{3} J_{0}^{\mu}+\frac{1}{2} J_{3}^{\mu}+\frac{1}{2 \sqrt{3}} J_{8}^{\mu}, \\
& J_{d}^{\mu}=\frac{1}{3} J_{0}^{\mu}-\frac{1}{2} J_{3}^{\mu}+\frac{1}{2 \sqrt{3}} J_{8}^{\mu}, \\
& J_{s}^{\mu}=\frac{1}{3} J_{0}^{\mu}-\frac{1}{\sqrt{3}} J_{8}^{\mu},
\end{aligned}
$$

where $J_{3}^{\mu}$ and $J_{8}^{\mu}$ are the $a=3$ and 8 components of the octet current, respectively. Using Eqs. (14), (15) and (16), the currents $J_{u}^{\mu}, J_{d}^{\mu}$ and $J_{s}^{\mu}$ can be written explicitly as

$$
\begin{aligned}
J_{u}^{\mu}= & 2 \bar{p} \gamma^{\mu} p+\bar{n} \gamma^{\mu} n+\bar{\Lambda} \gamma^{\mu} \Lambda+2 \bar{\Sigma}^{+} \gamma^{\mu} \Sigma^{+}+\bar{\Sigma}^{0} \gamma^{\mu} \Sigma^{0}-\frac{1}{2 f^{2}}\left(\bar{p} \gamma^{\mu} p \pi^{+} \pi^{-}+2 \bar{p} \gamma^{\mu} p K^{+} K^{-}\right)+3 \bar{\Delta}_{\alpha}^{++} \gamma^{\alpha \beta \mu} \Delta_{\beta}^{++}+2 \bar{\Delta}_{\alpha}^{+} \gamma^{\alpha \beta \mu} \Delta_{\beta}^{+} \\
& +\bar{\Delta}_{\alpha}^{0} \gamma^{\alpha \beta \mu} \Delta_{\beta}^{0}+2 \bar{\Sigma}_{\alpha}^{*+} \gamma^{\alpha \beta \mu} \Sigma_{\beta}^{*+}+\bar{\Sigma}_{\alpha}^{* 0} \gamma^{\alpha \beta \mu} \Sigma_{\beta}^{* 0}+i\left(\pi^{-} \partial^{\mu} \pi^{+}-\pi^{+} \partial^{\mu} \pi^{-}\right)+i\left(K^{-} \partial^{\mu} K^{+}-K^{+} \partial^{\mu} K^{-}\right) \\
& -\frac{i(D+F)}{\sqrt{2} f} \bar{p} \gamma^{\mu} \gamma^{5} n \pi^{+}+\frac{i(D+3 F)}{\sqrt{12} f} \bar{p} \gamma^{\mu} \gamma^{5} \Lambda K^{+}-\frac{i(D-F)}{2 f} \bar{p} \gamma^{\mu} \gamma^{5} \Sigma^{0} K^{+} \\
& +\frac{\mathcal{C}}{\sqrt{12} f}\left(i \sqrt{6} \bar{p} \Theta^{\mu \nu} \Delta_{\nu}^{++} \pi^{-}+i \sqrt{2} \bar{p} \Theta^{\mu \nu} \Delta_{\nu}^{0} \pi^{+}+i \bar{p} \Theta^{\mu \nu} \Sigma_{\nu}^{* 0} K^{+}+\mathrm{H} . \mathrm{c} .\right) \\
J_{d}^{\mu}= & \bar{p} \gamma^{\mu} p+2 \bar{n} \gamma^{\mu} n+2 \bar{\Sigma}^{-} \gamma^{\mu} \Sigma^{-}+\bar{\Sigma}^{0} \gamma^{\mu} \Sigma^{0}+\bar{\Lambda} \gamma^{\mu} \Lambda+\frac{1}{2 f^{2}}\left(\bar{p} \gamma^{\mu} p \pi^{+} \pi^{-}-\bar{p} \gamma^{\mu} p \bar{K}^{0} K^{0}\right)+\bar{\Delta}_{\alpha}^{+} \gamma^{\alpha \beta \mu} \Delta_{\beta}^{+}+2 \bar{\Delta}_{\alpha}^{0} \gamma^{\alpha \beta \mu} \Delta_{\beta}^{0} \\
& +3 \bar{\Delta}_{\alpha}^{-} \gamma^{\alpha \beta \mu} \Delta_{\beta}^{-}+\bar{\Sigma}_{\alpha}^{* 0} \gamma^{\alpha \beta \mu} \Sigma_{\beta}^{* 0}+2 \bar{\Sigma}_{\alpha}^{* 0-} \gamma^{\alpha \beta \mu} \Sigma_{\beta}^{*-}-i\left(\pi^{-} \partial^{\mu} \pi^{+}-\pi^{+} \partial^{\mu} \pi^{-}\right)+i\left(\bar{K}^{0} \partial^{\mu} K^{0}-K^{0} \partial^{\mu} \bar{K}^{0}\right) \\
& +\frac{i(D+F)}{\sqrt{2} f} \bar{p} \gamma^{\mu} \gamma^{5} n \pi^{+}-\frac{i(D-F)}{\sqrt{2} f} \bar{p} \gamma^{\mu} \gamma^{5} \Sigma^{+} K^{0}-\frac{\mathcal{C}}{\sqrt{6} f}\left(i \sqrt{3} \bar{p} \Theta^{\mu \nu} \Delta_{\nu}^{++} \pi^{-}+i \bar{p} \Theta^{\mu \nu} \Delta_{\nu}^{0} \pi^{+}+i \bar{p} \Theta^{\mu \nu} \Sigma_{\nu}^{*+} K^{0}+\text { H.c. }\right) \\
J_{s}^{\mu}= & \bar{\Sigma}^{+} \gamma^{\mu} \Sigma^{+}+\bar{\Sigma}^{0} \gamma^{\mu} \Sigma^{0}+\bar{\Lambda} \gamma^{\mu} \Lambda+\frac{1}{2 f^{2}}\left(2 \bar{p} \gamma^{\mu} p K^{+} K^{-}+\bar{p} \gamma^{\mu} p \bar{K}^{0} K^{0}\right)+\bar{\Sigma}_{\alpha}^{*+} \gamma^{\alpha \beta \mu} \Sigma_{\beta}^{*+}+\bar{\Sigma}_{\alpha}^{* 0} \gamma^{\alpha \beta \mu} \Sigma_{\beta}^{* 0} \\
& -i\left(K^{-} \partial^{\mu} K^{+}-K^{+} \partial^{\mu} K^{-}\right)-i\left(\bar{K}^{0} \partial^{\mu} K^{0}-K^{0} \partial^{\mu} \bar{K}^{0}\right)+\frac{i(D-F)}{\sqrt{2} f} \bar{p} \gamma^{\mu} \gamma^{5} \Sigma^{+} K^{0}+\frac{i(D-F)}{2 f} \bar{p} \gamma^{\mu} \gamma^{5} \Sigma^{0} K^{+} \\
& -\frac{i(D+3 F)}{\sqrt{12} f} \bar{p} \gamma^{\mu} \gamma^{5} \Lambda K^{+}+\frac{\mathcal{C}}{\sqrt{12} f}\left(-i \bar{p} \Theta^{\mu \nu} \Sigma_{\nu}^{* 0} K^{+}+i \sqrt{2} \bar{p} \Theta^{\mu \nu} \Sigma_{\nu}^{*+} K^{0}+\mathrm{H} . c .\right),
\end{aligned}
$$


where the terms involving the doubly-strange baryons $\Xi^{0,-}$ and $\Xi^{* 0,-}$ and the triply-strange $\Omega^{-}$are not present because they cannot couple to the proton initial states.

\section{NONLOCAL CHIRAL LAGRANGIAN}

In this section we describe the generation of the nonlocal Lagrangian from the local meson-baryon Lagrangian in Sec. II. Evaluating the traces in Eq. (1) and introducing the minimal substitution for the electromagnetic field $\mathcal{A}_{\mu}$, the local Lagrangian density can be rewritten more explicitly in the form

$$
\begin{aligned}
\mathcal{L}^{(\text {local })}(x)= & \bar{B}(x)\left(i \gamma^{\mu} \mathcal{D}_{\mu, x}-M_{B}\right) B(x)+\frac{C_{B \phi}}{f}\left[\bar{p}(x) \gamma^{\mu} \gamma^{5} B(x) \mathcal{D}_{\mu, x} \phi(x)+\text { H.c. }\right]+\bar{T}_{\mu}(x)\left(i \gamma^{\mu \nu \alpha} \mathcal{D}_{\alpha, x}-M_{T} \gamma^{\mu \nu}\right) T_{\nu}(x) \\
& +\frac{C_{T \phi}}{f}\left[\bar{p}(x) \Theta^{\mu \nu} T_{\nu}(x) \mathcal{D}_{\mu, x} \phi(x)+\text { H.c. }\right]+\frac{i C_{\phi \phi^{\dagger}}}{2 f^{2}} \bar{p}(x) \gamma^{\mu} p(x)\left[\phi(x)\left(\mathcal{D}_{\mu, x} \phi\right)^{\dagger}(x)-\mathcal{D}_{\mu, x} \phi(x) \phi^{\dagger}(x)\right] \\
& +\mathcal{D}_{\mu, x} \phi(x)\left(\mathcal{D}_{\mu, x} \phi\right)^{\dagger}(x)+\cdots,
\end{aligned}
$$

where for the interaction part we show only those terms that contribute to a meson-baryon coupling to a proton, and we keep the dependence on the space-time coordinate $x$ explicitly. The covariant derivatives here are written so as to indicate the coordinate with respect to which the derivative is taken,

$$
\begin{aligned}
\mathcal{D}_{\mu, x} B(x) & =\left[\partial_{\mu}-i e_{B}^{q} \mathcal{A}_{\mu}(x)\right] B(x), \\
\mathcal{D}_{\mu, x} T^{\nu}(x) & =\left[\partial_{\mu}-i e_{T}^{q} \mathcal{A}_{\mu}(x)\right] T^{\nu}(x), \\
\mathcal{D}_{\mu, x} \phi(x) & =\left[\partial_{\mu}-i e_{\phi}^{q} \mathcal{A}_{\mu}(x)\right] \phi(x),
\end{aligned}
$$

where $e_{B}^{q}, e_{T}^{q}$ and $e_{\phi}^{q}$ are the quark flavor charges of the octet baryon $B$, decuplet baryon $T$ and meson $\phi$, respectively. For example, for the proton one has the charges $e_{p}^{u}=2 e_{p}^{d}=2, e_{p}^{s}=0$, while for the $\Sigma^{+}$hyperon $e_{\Sigma^{+}}^{u}=2 e_{\Sigma^{+}}^{s}=2, e_{\Sigma^{+}}^{d}=0$, and so forth. For the mesons, the flavor charges for the $\pi^{+}$are $e_{\pi^{+}}^{u}=-e_{\pi^{+}}^{d}=1$ but $e_{\pi^{0}}^{q}=0$ for all $q$, and for the $K^{+}$these are $e_{K^{+}}^{u}=-e_{K^{+}}^{s}=1, e_{K^{+}}^{d}=0$, and similarly for the charge conjugate states. These flavor charges may be read off from the currents given in Eqs. (17a)-(17c). The coefficients $C_{B \phi}$ and $C_{T \phi}$ in Eq. (18) depend on the coupling constants $D, F$ and $\mathcal{C}$, and are given explicitly in Table I for the processes discussed in this work.

Using the methods described in Refs. [32,37-42], the nonlocal version of the local Lagrangian (18) can be written as

$$
\begin{aligned}
\mathcal{L}^{\text {(nonloc) }}(x)= & \bar{B}(x)\left(i \gamma^{\mu} \mathcal{D}_{\mu, x}-M_{B}\right) B(x)+\bar{T}_{\mu}(x)\left(i \gamma^{\mu \nu \alpha} \mathcal{D}_{\alpha, x}-M_{T} \gamma^{\mu \nu}\right) T_{\nu}(x) \\
& +\bar{p}(x)\left[\frac{C_{B \phi}}{f} \gamma^{\mu} \gamma^{5} B(x)+\frac{C_{T \phi}}{f} \Theta^{\mu \nu} T_{\nu}(x)\right] \int d^{4} a \mathcal{G}_{\phi}^{q}(x, x+a) F(a) \mathcal{D}_{\mu, x+a} \phi(x+a)+\text { H.c. } \\
& +\frac{i C_{\phi \phi^{\dagger}}}{2 f^{2}} \bar{p}(x) \gamma^{\mu} p(x) \int d^{4} a \int d^{4} b \mathcal{G}_{\phi}^{q}(x+b, x+a) F(a) F(b) \\
& \times\left[\phi(x+a)\left(\mathcal{D}_{\mu, x+b} \phi\right)^{\dagger}(x+b)-\mathcal{D}_{\mu, x+a} \phi(x+a) \phi^{\dagger}(x+b)\right]+\mathcal{D}_{\mu, x} \phi(x)\left(\mathcal{D}_{\mu, x} \phi\right)^{\dagger}(x)+\cdots,
\end{aligned}
$$

TABLE I. Coupling constants $C_{B \phi}, C_{T \phi}$ and $C_{\phi \phi^{\dagger}}$ for the $p B \phi, p T \phi$ and $p p \phi \phi^{\dagger}$ interactions, respectively, for the various allowed flavor channels.

\begin{tabular}{l|ccccc}
\hline \hline$(B \phi)$ & $\left(p \pi^{0}\right)$ & $\left(n \pi^{+}\right)$ & $\left(\Sigma^{+} K^{0}\right)$ & $\left(\Sigma^{0} K^{+}\right)$ & $\left(\Lambda K^{+}\right)$ \\
$C_{B \phi}$ & $\frac{1}{2}(D+F)$ & $\frac{1}{\sqrt{2}}(D+F)$ & $\frac{1}{\sqrt{2}}(D-F)$ & $\frac{1}{2}(D-F)$ & $-\frac{1}{\sqrt{12}}(D+3 F)$ \\
\hline$(T \phi)$ & $\left(\Delta^{0} \pi^{+}\right)$ & $\left(\Delta^{+} \pi^{0}\right)$ & $\left(\Delta^{++} \pi^{-}\right)$ & $\left(\Sigma^{*+} K^{0}\right)$ & $\left(\Sigma^{* 0} K^{+}\right)$ \\
$C_{T \phi}$ & $-\frac{1}{\sqrt{6}} \mathcal{C}$ & $-\frac{1}{\sqrt{3}} \mathcal{C}$ & $\frac{1}{\sqrt{2}} \mathcal{C}$ & $\frac{1}{\sqrt{6}} \mathcal{C}$ & $-\frac{1}{\sqrt{12}} \mathcal{C}$ \\
\hline$\left(\phi \phi^{\dagger}\right)$ & $\left(\pi^{+} \pi^{-}\right)$ & $\left(K^{0} \bar{K}^{0}\right)$ & $\left(K^{+} K^{-}\right)$ & & \\
$C_{\phi \phi^{\dagger}}$ & $\frac{1}{2}$ & $\frac{1}{2}$ & 1 & & \\
\hline \hline
\end{tabular}


where the gauge link $\mathcal{G}_{\phi}^{q}$ is introduced to preserve local gauge invariance,

$$
\mathcal{G}_{\phi}^{q}(x, y)=\exp \left[-i e_{\phi}^{q} \int_{x}^{y} d z^{\mu} \mathcal{A}_{\mu}(z)\right]
$$

and the function $F(a)$ is the meson-baryon vertex form factor in coordinate space. One can verify that the nonlocal Lagrangian in Eq. (20), as well as local Lagrangian in Eq. (18), are invariant under the gauge transformations

$$
\begin{aligned}
B(x) & \rightarrow B^{\prime}(x)=B(x) \exp \left[i e_{B}^{q} \theta(x)\right], \\
T_{\mu}(x) & \rightarrow T_{\mu}^{\prime}(x)=T_{\mu}(x) \exp \left[i e_{T}^{q} \theta(x)\right], \\
\phi(x) & \rightarrow \phi^{\prime}(x)=\phi(x) \exp \left[i e_{\phi}^{q} \theta(x)\right],
\end{aligned}
$$

for the matter fields, and

$$
\mathcal{A}^{\mu}(x) \rightarrow \mathcal{A}^{\prime \mu}(x)=\mathcal{A}^{\mu}(x)+\partial^{\mu} \theta(x)
$$

for the electromagnetic field, where $\theta(x)$ is an arbitrary function of the space-time coordinate $x^{\mu}$.

The nonlocal Lagrangian density in Eq. (20) can be further decomposed by expanding the gauge link (21) in powers of the charge $e_{\phi}^{q}$,

$$
\begin{aligned}
\mathcal{G}_{\phi}^{q}(x+b, x+a) \\
\quad=\exp \left[-i e_{\phi}^{q}(a-b)^{\mu} \int_{0}^{1} d t \mathcal{A}_{\mu}(x+a t+b(1-t))\right] \\
\quad=1+\delta \mathcal{G}_{\phi}^{q}+\cdots,
\end{aligned}
$$

where the $\mathcal{O}\left(e_{\phi}^{q}\right)$ term is

$\delta \mathcal{G}_{\phi}^{q}=-i e_{\phi}^{q}(a-b)^{\mu} \int_{0}^{1} d t \mathcal{A}_{\mu}(x+a t+b(1-t))$

and we have used a change of variables $z^{\mu} \rightarrow x^{\mu}+a^{\mu} t+$ $b^{\mu}(1-t)$. This allows the Lagrangian $\mathcal{L}^{(\text {nonloc) }}$ to be written as a sum of free and interacting parts, where to lowest order the latter consists of purely hadronic $\left(\mathcal{L}_{\text {had }}^{\text {(nonloc) }}\right)$, electromagnetic $\left(\mathcal{L}_{\text {em }}^{(\text {nonloc) }}\right)$, and gauge link $\left(\mathcal{L}_{\text {link }}^{\text {(nonloc) }}\right)$ components. The higher order terms in Eq. (23) contribute to higher order electromagnetic corrections, which are in practice negligible. The higher order terms can also be related to other processes, such as those involving two or more photons emitted in the final state.

The hadronic and electromagnetic interaction parts of the nonlocal Lagrangian arise from the $\mathcal{O}\left(e_{\phi}^{q}\right)$ term in Eq. (23), and are given by

$$
\begin{aligned}
\mathcal{L}_{\text {had }}^{\text {(nonloc) }}(x)= & \bar{p}(x)\left[\frac{C_{B \phi}}{f} \gamma^{\mu} \gamma^{5} B(x)+\frac{C_{T \phi}}{f} \Theta^{\mu \nu} T_{\nu}(x)\right] \int d^{4} a F(a) \partial_{\mu} \phi(x+a)+\text { H.c. } \\
& +\frac{i C_{\phi \phi^{\dagger}}}{2 f^{2}} \bar{p}(x) \gamma^{\mu} p(x) \int d^{4} a \int d^{4} b F(a) F(b)\left[\phi(x+a) \partial_{\mu} \phi^{\dagger}(x+b)-\partial_{\mu} \phi(x+a) \phi^{\dagger}(x+b)\right],
\end{aligned}
$$

and

$$
\begin{aligned}
\mathcal{L}_{\mathrm{em}}^{(\text {nonloc })}(x)= & e_{B}^{q} \bar{B}(x) \gamma^{\mu} B(x) \mathcal{A}_{\mu}(x)+e_{T}^{q} \bar{T}_{\mu}(x) \gamma^{\mu \nu \alpha} T_{\nu}(x) \mathcal{A}_{\alpha}(x)+i e_{\phi}^{q}\left[\partial^{\mu} \phi(x) \phi^{\dagger}(x)-\phi(x) \partial^{\mu} \phi^{\dagger}(x)\right] \mathcal{A}_{\mu}(x) \\
& -i e_{\phi}^{q} \bar{p}(x)\left[\frac{C_{B \phi}}{f} \gamma^{\mu} \gamma^{5} B(x)+\frac{C_{T \phi}}{f} \Theta^{\mu \nu} T_{\nu}(x)\right] \int d^{4} a F(a) \phi(x+a) \mathcal{A}^{\mu}(x+a)+\text { H.c. } \\
& -\frac{e_{\phi}^{q} C_{\phi \phi^{\dagger}}}{2 f^{2}} \bar{p}(x) \gamma^{\mu} p(x) \int d^{4} a F(a) \int d^{4} b F(b) \phi(x+a) \phi^{\dagger}(x+b)\left[\mathcal{A}^{\mu}(x+a)+\mathcal{A}^{\mu}(x+b)\right],
\end{aligned}
$$

respectively. For the $\delta \mathcal{G}_{\phi}^{q}$ term in Eq. (24), which explicitly depends on the gauge link, the nonlocal interaction with the external gauge field yields the additional contribution to the Lagrangian density,

$$
\begin{aligned}
\mathcal{L}_{\text {link }}^{\text {(nonloc) }}(x)= & -i e_{\phi}^{q} \bar{p}(x)\left[\frac{C_{B \phi}}{f} \gamma^{\rho} \gamma^{5} B(x)+\frac{C_{T \phi}}{f} \Theta^{\rho \nu} T_{\nu}(x)\right] \int_{0}^{1} d t \int d^{4} a F(a) a^{\mu} \partial_{\rho} \phi(x+a) \mathcal{A}_{\mu}(x+a t)+\text { H.c. } \\
& +\frac{e_{\phi}^{q} C_{\phi \phi^{\dagger}}}{2 f^{2}} \bar{p}(x) \gamma^{\rho} p(x) \int_{0}^{1} d t \int d^{4} a \int d^{4} b F(a) F(b)(a-b)^{\mu}\left[\phi(x+a) \partial_{\rho} \phi^{\dagger}(x+b)-\partial_{\rho} \phi(x+a) \phi^{\dagger}(x+b)\right] \\
& \times \mathcal{A}_{\mu}(x+a t+b(1-t)) .
\end{aligned}
$$


For the nonlocal theory the quark current has two contributions: the usual electromagnetic current, $J_{q, \mathrm{em}}^{\mu}$, obtained with minimal substitution from Eq. (26),

$$
\begin{aligned}
J_{q, \mathrm{em}}^{\mu}(x) \equiv & \frac{\delta \int d^{4} y \mathcal{L}_{\mathrm{em}}^{(\mathrm{nonloc})}(y)}{\delta \mathcal{A}_{\mu}(x)} \\
= & e_{B}^{q} \bar{B}(x) \gamma^{\mu} B(x)+e_{T}^{q} \bar{T}_{\alpha}(x) \gamma^{\alpha \nu \mu} T_{\nu}(x)+i e_{\phi}^{q}\left[\partial^{\mu} \phi(x) \phi^{\dagger}(x)-\phi(x) \partial^{\mu} \phi^{\dagger}(x)\right] \\
& -i e_{\phi}^{q} \int d^{4} a F(a) \bar{p}(x-a)\left[\frac{C_{B \phi}}{f} \gamma^{\mu} \gamma^{5} B(x-a)+\frac{C_{T \phi}}{f} \Theta^{\mu \nu} T_{\nu}(x-a)\right] \phi(x)+\text { H.c. } \\
& -\frac{e_{\phi}^{q} C_{\phi \phi^{\dagger}}}{2 f^{2}} \int d^{4} a F(a) \int d^{4} b F(b)\left[\bar{p}(x-a) \gamma^{\mu} p(x-a) \phi(x) \phi^{\dagger}(x+b-a)\right. \\
& \left.+\bar{p}(x-b) \gamma^{\mu} p(x-b) \phi(x+a-b) \phi^{\dagger}(x)\right],
\end{aligned}
$$

and an additional term obtained from the gauge link,

$$
\begin{aligned}
\delta J_{q}^{\mu}(x) \equiv & \frac{\delta \int d^{4} y \mathcal{L}_{\text {link }}^{(\text {nonloc })}(y)}{\delta \mathcal{A}_{\mu}(x)} \\
= & -i e_{\phi}^{q} \int_{0}^{1} d t \int d^{4} a F(a) a^{\mu} \bar{p}(x-a t)\left[\frac{C_{B \phi}}{f} \gamma^{\rho} \gamma^{5} B(x-a t)+\frac{C_{T \phi}}{f} \Theta^{\rho \nu} T_{\nu}(x-a t)\right] \partial_{\rho} \phi(x+a(1-t))+\text { H.c. } \\
& +\frac{e_{\phi}^{q} C_{\phi \phi^{\dagger}}}{2 f^{2}} \int_{0}^{1} d t \int d^{4} a F(a) \int d^{4} b F(b)(a-b)^{\mu} \bar{p}(x-a t-b(1-t)) \gamma^{\rho} p(x-a t-b(1-t)) \\
& \times\left[\phi(x+(a-b)(1-t)) \partial_{\rho} \phi^{\dagger}(x-(a-b) t)-\partial_{\rho} \phi(x+(a-b)(1-t)) \phi^{\dagger}(x-(a-b) t)\right]
\end{aligned}
$$

respectively. Compared with Eqs. (13) and (17), the nonlocal interaction Lagrangian and currents in Eqs. (25)-(29) include the extra regulator function $F(a)$. The local limit can be obtained by taking $F(a)$ to be a $\delta$-function, $F(a) \rightarrow \delta^{(4)}(a)$, which is equivalent to taking the form factor in momentum space to be unity. Since the Fourier transform of the $\delta$-function in position space is a plane wave in momentum space, the value of the plane wave at the origin is unity.

Note that compared with traditional power counting schemes in chiral perturbation theory that use dimensional regularization [48], the introduction of the regulator function $F(a)$ in the nonlocal interactions (25)-(27) leads to the generation of higher order terms in $m_{\phi}$ with coefficients that in general will depend on the regulator mass, such as the large momentum cutoff parameter $\Lambda$ (see Sec. IV B below). This is analogous to a resummation of the standard chiral perturbation theory, which goes beyond the usual power counting regime, at the expense of introducing model dependence into the calculation. An advantage of this resummed approach is that one can obtain better convergence in $m_{\phi}$ in regions where the usual power counting schemes would not be applicable (see Refs. [49,50]).

\section{SPLITTING FUNCTIONS}

With the nonlocal interaction and current derived in Sec. III, in this section we will discuss the splitting functions describing the interaction of the external field with the proton dressed by the pseudoscalar fields. We will derive the general expressions for the proton $\rightarrow$ pseudoscalar meson + baryon splitting functions for the full set of SU(3) octet and decuplet states. After giving the general results for an arbitrary regulating function $F(a)$, we derive explicit expressions for a specific choice of regulator in which the momentum dependence is given by a dipole shape.

\section{A. Model independent results}

The interaction of an external probe with a proton dressed by pseudoscalar mesons at leading order is given in Fig. 1 for octet intermediate states and in Fig. 1 for decuplet intermediate states. The diagrams in Figs. 1(a)-(c), (e), (f), (h)-(j) correspond to those in the local effective theory, while those in Figs. 1(d), (g) and (k) arise from the new interactions in the nonlocal theory given by Eqs. (25)-(27). The resulting amplitudes will be expressed in terms of specific mesonbaryon splitting functions convoluted with corresponding PDFs in the bare or undressed mesons and baryons. These will be used to compute the contributions from meson loops to PDFs in the nucleon, the most direct predictions for which will be for nonsinglet PDF combinations in which perturbative QCD effects largely cancel. Examples include the light-antiquark flavor asymmetry $\bar{d}-\bar{u}$ and the strange asymmetry $s-\bar{s}$. In the valence approximation for the 


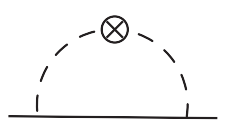

(a)

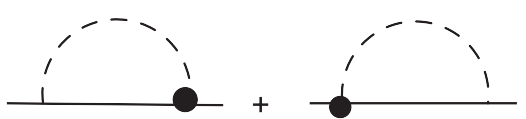

(d)

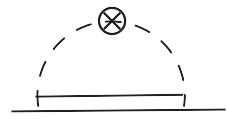

(h)

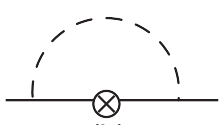

(b)

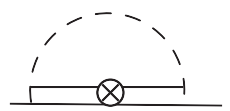

(i)

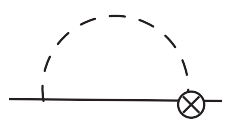

(c)

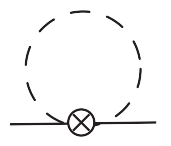

(e)

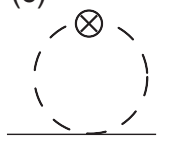

(f)
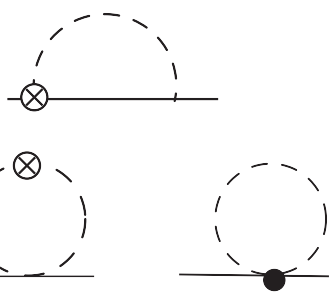

(g)

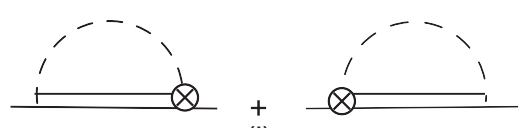

(j)

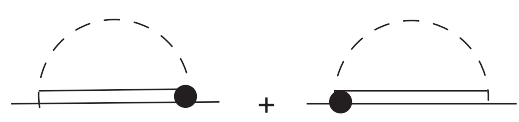

(k)

FIG. 1. Diagrams representing the interaction of an external current (denoted by the crossed circles) with the proton involving SU(3) octet [(a)-(g)] and decuplet [(h)-(j)] states: (a) and (h) are for meson coupling rainbow diagrams; (b) and (i) are for octet and decuplet baryon coupling rainbow diagrams; (c) and (k) are for Kroll-Ruderman; (d) and (j) are for Kroll-Ruderman type diagrams generated by the gauge link (denoted by the filled circle); (e) is for meson tadpole; (f) is for meson bubble; and (g) is for meson tadpole diagram generated by the gauge link.

undressed hadrons, the former will only receive contributions from the direct meson coupling diagrams in Figs. 1(a), (f) and (h), while all the diagrams in Fig. 1 will be relevant for the $s-\bar{s}$ asymmetry.

\section{1. $S U(3)$ octet intermediate states}

Beginning with the meson rainbow diagram in Fig. 1(a), the vertex function for the nonlocal theory can be written as [51]

$$
\begin{aligned}
\Gamma_{\phi B}^{\mu}(2 \pi)^{4} \delta^{(4)}(p-p)= & \left\langle p\left|i^{2} \int d^{4} x d^{4} y d^{4} z \mathcal{L}_{\mathrm{had}(B)}^{\text {(nonloc) }}(x) J_{\mathrm{q}, \mathrm{em}}^{\mu}(y) \mathcal{L}_{\mathrm{had}(B)}^{(\text {nonloc })}(z)\right| p\right\rangle \\
= & \frac{i^{2} C_{B \phi}^{2}}{f^{2}}\langle p| \int d^{4} x d^{4} y d^{4} z \int d^{4} a F(a) \int d^{4} b F(b) \bar{p}(x) \gamma^{\nu} \gamma^{5} B(x) \partial_{\nu} \phi(x+a) \\
& \times\left(-i\left[\phi(y) \partial^{\mu} \phi^{\dagger}(y)-\phi^{\dagger}(y) \partial^{\mu} \phi(y)\right]\right) \bar{B}(z) \gamma^{\rho} \gamma^{5} p(z) \partial_{\rho} \phi^{\dagger}(z+b)|p\rangle
\end{aligned}
$$

where $\mathcal{L}_{\mathrm{had}(B)}^{\text {(nonloc) }}$ is the part of the hadronic nonlocal Lagrangian (25) that depends on the octet baryon fields $B$. (Note also that we defined the vertex such that the quark flavor charge $e_{\phi}^{q}$ is included explicitly in the bare meson and baryon PDFs discussed in the next section.) Integrating over the space-time coordinates $x^{\mu}, y^{\mu}$ and $z^{\mu}$, one has

$\Gamma_{\phi B}^{\mu}=\frac{C_{B \phi}^{2}}{f^{2}} \bar{u}(p) \int \frac{d^{4} k}{(2 \pi)^{4}} \int d^{4} a F(a) \int d^{4} b F(b)\left(\not k \gamma^{5}\right) \frac{i\left[(\not p-\not k)+M_{B}\right]}{D_{B}}\left(\gamma^{5} \not k\right) \frac{i}{D_{\phi}} 2 k^{\mu} \frac{i}{D_{\phi}} u(p) \exp [-i k \cdot(a-b)]$,

where the Dirac spinor $u$ is normalized such that $\bar{u} u=1$, and $D_{\phi}$ and $D_{B}$ denote the propagator factors for the intermediate baryon and meson, respectively,

$$
\begin{aligned}
& D_{\phi}=k^{2}-m_{\phi}^{2}+i \varepsilon, \\
& D_{B}=(p-k)^{2}-M_{B}^{2}+i \varepsilon,
\end{aligned}
$$

where $m_{\phi}$ and $M_{B}$ are for the meson and octet baryon masses. Defining the regulator in momentum space as

$$
\widetilde{F}(k) \equiv \int d^{4} a \exp [-i a \cdot k] F(a)
$$

the vertex operator becomes 


$$
\begin{aligned}
\boldsymbol{\Gamma}_{\phi B}^{\mu} & =\frac{C_{B \phi}^{2}}{f^{2}} \bar{u}(p) \int \frac{d^{4} k}{(2 \pi)^{4}}\left(\not k \gamma^{5}\right) \widetilde{F}(k) \frac{i\left[(\not p-\not k)+M_{B}\right]}{D_{B}} \frac{i}{D_{\phi}} 2 k^{\mu} \frac{i}{D_{\phi}}\left(\gamma^{5} \not k\right) \widetilde{F}(-k) u(p) \\
& \equiv \int \frac{d^{4} k}{(2 \pi)^{4}} \tilde{\boldsymbol{\Gamma}}_{\phi B}^{\mu} .
\end{aligned}
$$

Taking the $\mu=+$ component of the integrand $\tilde{\boldsymbol{\Gamma}}_{\phi B}^{\mu}$, we define the splitting function $f_{\phi B}^{(\mathrm{rbw})}(y)$ in terms of the light-cone projection of $\tilde{\boldsymbol{\Gamma}}_{\phi B}^{\mu}$,

$$
f_{\phi B}^{(\mathrm{rbw})}(y)=\frac{M}{p^{+}} \int \frac{d^{4} k}{(2 \pi)^{4}} \tilde{\boldsymbol{\Gamma}}_{\phi B}^{+} \delta\left(y-\frac{k^{+}}{p^{+}}\right)
$$

where $k^{+}=k^{0}+k^{z}$ and $M$ is the nucleon mass. From Eq. (34) the splitting function for the meson rainbow diagram is then given by

$$
f_{\phi B}^{(\mathrm{rbw})}(y)=\frac{C_{B \phi}^{2}}{f^{2}} \int \frac{d^{4} k}{(2 \pi)^{4}} \bar{u}(p)\left(\not \gamma^{5}\right) \frac{i\left[(\not p-\not k)+M_{B}\right]}{D_{B}} \frac{i}{D_{\phi}}\left(2 k^{+}\right) \frac{i}{D_{\phi}}\left(\gamma^{5} \not k\right) \widetilde{F}^{2}(k) u(p) \frac{M}{p^{+}} \delta\left(y-\frac{k^{+}}{p^{+}}\right) .
$$

Similarly, the splitting functions for the baryon rainbow diagram of Fig. 1(b) and the Kroll-Ruderman (KR) diagram of Fig. 1(c) can be expressed as

$$
f_{B \phi}^{(\mathrm{rbw})}(y)=\frac{C_{B \phi}^{2}}{f^{2}} \int \frac{d^{4} k}{(2 \pi)^{4}} \bar{u}(p)\left(\not k \gamma^{5}\right) \frac{i\left[(\not p-\not k)+M_{B}\right]}{D_{B}} \gamma^{+} \frac{i\left[(\not p-\not k)+M_{B}\right]}{D_{B}}\left(\gamma^{5} \not k\right) \frac{i}{D_{\phi}} \widetilde{F}^{2}(k) u(p) \frac{M}{p^{+}} \delta\left(y-\frac{k^{+}}{p^{+}}\right)
$$

and

$$
\begin{aligned}
f_{B}^{(\mathrm{KR})}(y)= & \frac{C_{B \phi}^{2}}{f^{2}} \int \frac{d^{4} k}{(2 \pi)^{4}} \bar{u}(p)\left\{\left(i \gamma^{+} \gamma^{5}\right) \frac{i\left[(\not p-\not k)+M_{B}\right]}{D_{B}}\left(\gamma^{5} \not k\right)+\left(\not k \gamma^{5}\right) \frac{i\left[(\not p-\not k)+M_{B}\right]}{D_{B}}\left(i \gamma^{5} \gamma^{+}\right)\right\} \\
& \times \frac{i}{D_{\phi}} \widetilde{F}^{2}(k) u(p) \frac{M}{p^{+}} \delta\left(y-\frac{k^{+}}{p^{+}}\right)
\end{aligned}
$$

respectively.

As discussed in Sec. III, the current generated by the gauge link in Eq. (29) produces the additional diagrams in Fig. 1(d), 1(g) and 1(k). The amplitude for the Kroll-Ruderman additional diagram in Fig. 1(d) can be written as

$$
\begin{aligned}
\delta \Gamma_{B}^{\mu}(2 \pi)^{4} \delta^{(4)}(p-p)= & \langle p| i \int d^{4} y d^{4} z\left(\mathcal{L}_{\mathrm{had}(B)}^{(\text {nonloc })}(y) \delta J_{q}^{\mu}(z)+\delta J_{q}^{\mu}(y) \mathcal{L}_{\mathrm{had}(B)}^{(\mathrm{nonloc})}(z)\right) \\
= & \frac{i C_{B \phi}^{2}}{f^{2}} \int_{0}^{1} d t\langle p| \int d^{4} y d^{4} z \int d^{4} a F(a) \int d^{4} b F(b) \\
& \times\left[-i b^{\mu} \bar{p}(y) \gamma^{\nu} \gamma^{5} B(y) \partial_{\nu} \phi(y+a) \bar{B}(z-b t) \gamma^{\rho} \gamma^{5} p(z-b t) \partial_{\rho} \phi^{\dagger}(z+b(1-t))\right. \\
& \left.+i a^{\mu} \bar{p}(y-a t) \gamma^{\nu} \gamma^{5} B(y-a t) \partial_{\nu} \phi(y+t(1-a)) \bar{B}(z) \gamma^{\rho} \gamma^{5} p(z) \partial_{\rho} \phi^{\dagger}(z+b)\right]|p\rangle,
\end{aligned}
$$

which after Wick contraction and integration over $x^{\mu}, y^{\mu}$ and $z^{\mu}$, becomes

$$
\begin{aligned}
\delta \Gamma_{B}^{\mu}= & \frac{i C_{B \phi}^{2}}{f^{2}} \bar{u}(p) \int d^{4} a F(a) \int d^{4} b F(b) \int \frac{d^{4} k}{(2 \pi)^{4}} \\
& \times\left\{-i b^{\mu}\left(\not k \gamma^{5}\right) \frac{i\left[(\not p-\not k)+M_{B}\right]}{D_{B}}\left(\not k \gamma^{5}\right) \frac{i}{D_{\phi}}+i a^{\mu}\left(\not k \gamma^{5}\right) \frac{i}{D_{\phi}} \frac{i\left[(\not p-\not k)+M_{B}\right]}{D_{B}}\left(\not k \gamma^{5}\right)\right\} u(p) \exp [-i k \cdot(a-b)] .
\end{aligned}
$$

Performing the integrations over the space-time coordinates $a^{\mu}$ and $b^{\mu}$, the vertex can be further simplified to 


$$
\begin{aligned}
\delta \boldsymbol{\Gamma}_{B}^{\mu}= & \frac{i C_{B \phi}^{2}}{f^{2}} \bar{u}(p) \int \frac{d^{4} k}{(2 \pi)^{4}}\left\{-\frac{\partial \widetilde{F}(-k)}{\partial k^{\mu}} \widetilde{F}(k)\left(\not k \gamma^{5}\right) \frac{i\left[(\not p-\not k)+M_{B}\right]}{D_{B}}\left(\not k \gamma^{5}\right)\right. \\
& \left.-\frac{\partial \widetilde{F}(k)}{\partial k^{\mu}} \widetilde{F}(-k)\left(\not k \gamma^{5}\right) \frac{i\left[(\not p-\not k)+M_{B}\right]}{D_{B}}\left(\not k \gamma^{5}\right)\right\} \frac{i}{D_{\phi}} u(p) .
\end{aligned}
$$

In analogy with the definition of the splitting function in Eq. (34), the splitting function for the nonlocal Kroll-Ruderman diagram in Fig. 1(d) induced by the gauge link can be written as

$$
\delta f_{B}^{(\mathrm{KR})}(y)=\frac{2 C_{B \phi}^{2}}{f^{2}} \int \frac{d^{4} k}{(2 \pi)^{4}} \bar{u}(p)\left(i \not k \gamma^{5}\right) \frac{i\left[(\not p-\not k)+M_{B}\right]}{D_{B}} \frac{i}{D_{\phi}}\left(-\not k \gamma^{5}\right) u(p) \frac{\partial \widetilde{F}^{2}(k)}{\partial k^{-}} \frac{M}{p^{+}} \delta\left(y-\frac{k^{+}}{p^{+}}\right) .
$$

The main additional feature here compared with the splitting functions in the local theory is the dependence on the derivative of the hadronic form factor $\tilde{F}$ on $k^{-}$.

For the remaining meson tadpole and bubble diagrams in Figs. 1(e) and 1(f), the splitting functions are given by

$$
f_{\phi}^{(\mathrm{tad})}(y)=\frac{C_{\phi \phi^{\dagger}}}{f^{2}} \int \frac{d^{4} k}{(2 \pi)^{4}} \bar{u}(p) \gamma^{+} \frac{i}{D_{\phi}} u(p) \widetilde{F}^{2}(k) \frac{M}{p^{+}} \delta\left(y-\frac{k^{+}}{p^{+}}\right),
$$

and

$$
f_{\phi}^{(\mathrm{bub})}(y)=-\frac{i C_{\phi \phi^{\dagger}}}{f^{2}} \int \frac{d^{4} k}{(2 \pi)^{4}} \bar{u}(p) 2 \not k k^{+}\left(\frac{i}{D_{\phi}}\right)^{2} u(p) \widetilde{F}^{2}(k) \frac{M}{p^{+}} \delta\left(y-\frac{k^{+}}{p^{+}}\right),
$$

where the coupling constant $C_{\phi \phi^{\dagger}}$ is listed in Table I.

Finally, the vertex associated with the nonlocal tadpole diagram in Fig. 1(g), generated by the gauge link, is defined by

$$
\delta \Gamma_{\phi}^{\mu}(2 \pi)^{4} \delta^{(4)}\left(p^{\prime}-p\right)=\left\langle p^{\prime}\left|\int d^{4} x \delta J_{q}^{\mu}(x)\right| p\right\rangle
$$

and can be reduced to

$$
\delta \boldsymbol{\Gamma}_{\phi}^{\mu}=\frac{C_{\phi \phi^{\dagger}}}{f^{2}} \int \frac{d^{4} k}{(2 \pi)^{4}} \bar{u}(p) \not k \frac{i}{D_{\phi}} u(p)\left[\widetilde{F}(-k) \frac{\partial \widetilde{F}(k)}{\partial k^{\mu}}+\widetilde{F}(k) \frac{\partial \widetilde{F}(-k)}{\partial k^{\mu}}\right] .
$$

The splitting function for the nonlocal tadpole diagram is then given by

$$
\delta f_{\phi}^{(\mathrm{tad})}(y)=\frac{C_{\phi \phi^{\dagger}}}{f^{2}} \int \frac{d^{4} k}{(2 \pi)^{4}} \bar{u}(p) \not k \frac{i}{D_{\phi}} u(p) \frac{2 \partial \widetilde{F}^{2}(k)}{\partial k^{-}} \frac{M}{p^{+}} \delta\left(y-\frac{k^{+}}{p^{+}}\right) .
$$

\section{Decuplet intermediate states}

For the splitting functions associated with the decuplet intermediate states in Fig. 1, the diagrams in Figs. 1(h), 1(i) and 1(j) arising from the local Lagrangian are supplemented by the additional nonlocal Kroll-Ruderman diagram in Fig. 1(k) induced by the gauge link in the nonlocal theory. Similarly to the meson rainbow contribution in Eq. (30), the vertex function for the meson rainbow diagram in Fig. 1(h) with an intermediate decuplet baryon $T$ can be written

$$
\begin{aligned}
\Gamma_{\phi T}^{\mu}(2 \pi)^{4} \delta^{(4)}(p-p)= & \left\langle p\left|i^{2} \int d^{4} x d^{4} y d^{4} z \mathcal{L}_{\text {had }(T)}^{(\text {nonloc }}(x) J_{q, \text { em }}^{\mu}(y) \mathcal{L}_{\text {had }(T)}^{(\text {nonloc })}(z)\right| p\right\rangle \\
= & \frac{i^{2} C_{T \phi}^{2}}{f^{2}}\langle p| \int d^{4} x d^{4} y d^{4} z \int d^{4} a F(a) \int d^{4} b F(b) \bar{p}(x) \Theta^{\alpha \beta} T_{\beta}(x) \partial_{\alpha} \phi(x+a) \\
& \times\left\{-i\left[\phi(y) \partial^{\mu} \phi^{\dagger}(y)-\phi^{\dagger}(y) \partial^{\mu} \phi(y)\right]\right\} \bar{T}_{\rho}(z) \Theta^{\rho \sigma} p(z) \partial_{\sigma} \phi^{\dagger}(z+b)|p\rangle,
\end{aligned}
$$


where $\mathcal{L}_{\operatorname{had}(T)}^{(\text {nonlo })}$ is the part of the hadronic nonlocal Lagrangian (25) that depends on the decuplet baryon fields $T$, and the operator $\Theta^{\alpha \beta}$ is given in Eq. (2). Integrating over the space-time coordinates, one finds

$$
\begin{aligned}
\Gamma_{\phi T}^{\mu}= & \frac{i^{2} C_{T \phi}^{2}}{f^{2}} \bar{u}(p) \int \frac{d^{4} k}{(2 \pi)^{4}} \int d^{4} b F(b) \int d^{4} a F(a) k_{\alpha} \Theta^{\alpha \beta} \frac{-i\left[(\not p-\not k)+M_{T}\right] P_{\beta \rho}(p-k)}{D_{T}} \\
& \times \frac{i}{D_{\phi}} 2 k^{\mu} \frac{i}{D_{\phi}} \Theta^{\rho \sigma} k_{\sigma} u(p) \exp [-i k \cdot(a-b)],
\end{aligned}
$$

where the decuplet baryon propagator $D_{T}$ is the same as $D_{B}$ in Eq. (32b), but with $M_{B}$ replaced by decuplet baryon mass $M_{T}$. The spin-3/2 projection operator $P_{\alpha \beta}$, like the octet-decuplet vertex function $\Theta_{\alpha \beta}$, depends on the off-shell parameter $Z$, defined in Eq. (2). However, as physical quantities do not depend on $Z$, it makes sense to simplify the form of the spin-3/2 propagator, and hence in our calculation we choose $Z=1 / 2$, following Refs. [52,53], in which case the projector $P_{\alpha \beta}$ is written

$$
P_{\alpha \beta}(p)=g_{\alpha \beta}-\frac{1}{3} \gamma_{\alpha} \gamma_{\beta}-\frac{\gamma_{\alpha} p_{\beta}-\gamma_{\beta} p_{\alpha}}{3 M_{T}}-\frac{2 p_{\alpha} p_{\beta}}{3 M_{T}^{2}}
$$

Note that for this choice one then has the operator $\Theta^{\alpha \beta}=g^{\alpha \beta}-\gamma^{\alpha} \gamma^{\beta}$. Performing the integrations over the space-time coordinates $a^{\mu}$ and $b^{\mu}$ then gives

$$
\boldsymbol{\Gamma}_{\phi T}^{\mu}=\frac{i^{2} C_{T \phi}^{2}}{f^{2}} \bar{u}(p) \int \frac{d^{4} k}{(2 \pi)^{4}} k_{\alpha} \Theta^{\alpha \beta} \widetilde{F}(k) \frac{-i\left[(\not p-\not k)+M_{T}\right] P_{\beta \rho}(p-k)}{D_{T}} \frac{i}{D_{\phi}} 2 k^{\mu} \frac{i}{D_{\phi}} \Theta^{\rho \sigma} k_{\sigma} \widetilde{F}(-k) u(p) .
$$

The splitting function for the meson rainbow diagram with decuplet intermediate state is therefore given by

$$
\begin{aligned}
f_{\phi T}^{(\mathrm{rbw})}(y)= & \frac{C_{T \phi}^{2}}{f^{2}} \int \frac{d^{4} k}{(2 \pi)^{4}} \bar{u}(p) k_{\alpha} \Theta^{\alpha \beta} \frac{-i\left[(\not p-\not k)+M_{T}\right] P_{\beta \rho}(p-k)}{D_{T}} \\
& \times \frac{i}{D_{\phi}} 2 k^{+} \frac{i}{D_{\phi}}\left(-\Theta^{\rho \sigma} k_{\sigma}\right) u(p) \widetilde{F}^{2}(k) \frac{M}{p^{+}} \delta\left(y-\frac{k^{+}}{p^{+}}\right) .
\end{aligned}
$$

Following similar procedures as for the octet baryon case, the splitting functions for the decuplet baryon rainbow diagram in Fig. 1(i) and the decuplet Kroll-Ruderman diagram in Fig. 1(j) can be written as

$$
\begin{aligned}
& f_{T \phi}^{(\mathrm{rbw})}(y)=\frac{C_{T \phi}^{2}}{f^{2}} \int \frac{d^{4} k}{(2 \pi)^{4}} \bar{u}(p) k_{\mu} \Theta^{\mu \nu} \frac{-i\left[(\not p-\not k)+M_{T}\right] P_{\nu \alpha}(p-k)}{D_{T}} \gamma^{\alpha \beta+} \\
& \times \frac{-i\left[(\not p-\not k)+M_{T}\right] P_{\beta \rho}(p-k)}{D_{T}} \frac{i}{D_{\phi}}\left(-\Theta^{\rho \sigma} k_{\sigma}\right) u(p) \widetilde{F}^{2}(k) \frac{M}{p^{+}} \delta\left(y-\frac{k^{+}}{p^{+}}\right)
\end{aligned}
$$

and

$$
\begin{aligned}
f_{T}^{(\mathrm{KR})}(y) & =\frac{C_{T \phi}^{2}}{f^{2}} \int \frac{d^{4} k}{(2 \pi)^{4}} \bar{u}(p)\left\{\frac{i}{D_{\phi}}\left(i \Theta^{+\nu}\right) \frac{-i\left[(\not p-\not k)+M_{T}\right] P_{\nu \alpha}(p-k)}{D_{T}}\left(-\Theta^{\alpha \sigma} k_{\sigma}\right)\right. \\
& \left.+k_{\mu} \Theta^{\mu \nu} \frac{-i\left[(\not p-\not k)+M_{T}\right] P_{\nu \alpha}(p-k)}{D_{T}}\left(-i \Theta^{\alpha+}\right) \frac{i}{D_{\phi}}\right\} u(p) \widetilde{F}^{2}(k) \frac{M}{p^{+}} \delta\left(y-\frac{k^{+}}{p^{+}}\right),
\end{aligned}
$$

respectively. Finally, the splitting function for the nonlocal Kroll-Ruderman decuplet diagram in Fig. 1(k) induced by the gauge link is

$$
\delta f_{T}^{(\mathrm{KR})}(y)=\frac{2 C_{T \phi}^{2}}{f^{2}} \int \frac{d^{4} k}{(2 \pi)^{4}} \bar{u}(p)\left(i k_{\sigma} \Theta^{\sigma \nu}\right) \frac{-i\left[(\not p-\not k)+M_{T}\right] P_{\nu \alpha}(p-k)}{D_{T}} \frac{i}{D_{\phi}}\left(-\Theta^{\alpha \sigma} k_{\sigma}\right) u(p) \frac{\partial \widetilde{F}^{2}(k)}{\partial k^{-}} \frac{M}{p^{+}} \delta\left(y-\frac{k^{+}}{p^{+}}\right) .
$$


The set of functions $\left\{f_{\phi B}^{(\mathrm{rbw})}, f_{B \phi}^{(\mathrm{rbw})}, f_{B}^{(\mathrm{KR})}, \delta f_{B}^{(\mathrm{KR})}, f_{\phi}^{(\mathrm{bub})}\right.$, $\left.f_{\phi}^{(\mathrm{tad})}, \delta f_{\phi}^{(\mathrm{tad})}\right\}$ for the octet baryons, and $\left\{f_{\phi T}^{(\mathrm{rbw})}, f_{T \phi}^{(\mathrm{rbw})}\right.$, $\left.f_{T}^{(\mathrm{KR})}, \delta f_{T}^{(\mathrm{KR})}\right\}$ for the decuplet baryons, then represent the complete set of functions that describe the dressing at one loop of the interaction of an external current with the proton in the nonlocal meson-baryon field theory.

\section{B. Covariant dipole form factor}

To evaluate the splitting functions derived in the previous section requires a specific choice for the meson-baryon vertex form factor $\tilde{F}(k)$. Consistency with Lorentz invariance restricts the form factor to in general be a function of the meson virtuality $k^{2}$ and the baryon virtuality $(p-k)^{2}$. For illustration, we choose the regulator to have a simple dipole shape in $k^{2}$ with a cutoff parameter $\Lambda$ [35,36], independent of the details of the baryon state,

$$
\widetilde{F}(k)=\frac{\bar{\Lambda}^{4}}{D_{\Lambda}^{2}},
$$

where $D_{\Lambda}=k^{2}-\Lambda^{2}+i \varepsilon$ and we define $\bar{\Lambda}^{2} \equiv \Lambda^{2}-m_{\phi}^{2}$. Other forms, such as Guassian, monopole or sharp cutoff, have also been used in the literature [49,50], and, with appropriate choices of regulator mass for the different regulators, give rise to qualitatively similar results. An advantage of the dipole form (56) is that it allows a more direct comparison with previous literature $[35,36,54]$ that has used the same functional form.

\section{Octet splitting functions}

With the dipole regulator in Eq. (56), after reduction of the $\gamma$ matrices in Eq. (36) the splitting function for the meson rainbow diagram in Fig. 1(a) can be written as

$$
\begin{aligned}
f_{\phi B}^{(\mathrm{rbw})}(y)= & \frac{i C_{B \phi}^{2} \bar{\Lambda}^{8}}{f^{2}} \int \frac{d^{4} k}{(2 \pi)^{4}}\left[\frac{y \bar{M}^{2}\left(\Delta^{2}-m_{\phi}^{2}\right)}{D_{\phi}^{2} D_{B} D_{\Lambda}^{4}}-\frac{y \bar{M}^{2}}{D_{\phi} D_{B} D_{\Lambda}^{4}}\right. \\
& \left.+\frac{y(\bar{M} \Delta-2 p \cdot k)}{D_{\phi}^{2} D_{\Lambda}^{4}}\right] \delta\left(y-\frac{k^{+}}{p^{+}}\right),
\end{aligned}
$$

where the average mass $\bar{M}$ and mass difference $\Delta$ are defined as

$$
\bar{M}=M+M_{B}, \quad \Delta=M_{B}-M .
$$

It will be convenient to perform the $d^{4} k$ integration in terms of light-cone momentum components $k^{ \pm}=k^{0} \pm k^{z}$ and transverse momentum $\boldsymbol{k}_{\perp}$. The first two terms in Eq. (57) have poles both on the upper and lower half-plane, so the integration over $k^{-}$can be obtained using the residue of $D_{B}$ or $D_{\phi}$. For the third term, proportional to $1 / D_{\phi}^{2}$, when $k^{+} \neq 0$ both $D_{\phi}$ and $D_{\Lambda}$ have poles on same half-plane, so the integral vanishes. On the other hand, when $k^{+}=0$ the integral becomes divergent. We can simplify this term using

$$
\begin{aligned}
\int d^{4} k \frac{2 y p \cdot k}{D_{\phi}^{2} D_{\Lambda}^{4}} & =\frac{\partial^{4}}{6 \partial \Omega^{4}} \int_{0}^{1} d z \int d^{4} k \frac{2 p \cdot k y(1-z) z^{3}}{\left(k^{2}-\Omega+i \varepsilon\right)^{2}} \\
& =\frac{\partial^{4}}{6 \partial \Omega^{4}} \int_{0}^{1} d z \int d^{4} k \frac{(1-z) z^{3}}{\left(k^{2}-\Omega+i \varepsilon\right)},
\end{aligned}
$$

where we define

$$
\Omega \equiv(1-z) m_{\phi}^{2}+z \Lambda^{2} .
$$

The integration over $k^{-}$in Eq. (59) can be written as [24,55]

$$
\int_{-\infty}^{\infty} d k^{-} \frac{1}{k^{2}-\Omega+i \varepsilon}=2 \pi i \log \left(\frac{k_{\perp}^{2}+\Omega}{\mu^{2}}\right) \delta\left(k^{+}\right),
$$

where $\mu$ is a momentum independent constant. After the $k^{-}$ integration, the splitting function for the meson rainbow diagram can be expressed as a sum of an on-shell term, $f_{B}^{(\text {on })}$, and $\delta$-function terms, $f_{\phi}^{(\delta)}$ and $\delta f_{\phi}^{(\delta)}$, generated by the contact interaction,

$f_{\phi B}^{(\mathrm{rbw})}(y)=\frac{C_{B \phi}^{2} \bar{M}^{2}}{(4 \pi f)^{2}}\left[f_{B}^{(\mathrm{on})}(y)+f_{\phi}^{(\delta)}(y)-\delta f_{\phi}^{(\delta)}(y)\right]$.

The on-shell function is given by

$$
f_{B}^{(\text {on })}(y)=\bar{\Lambda}^{8} \int d k_{\perp}^{2} \frac{y\left[k_{\perp}^{2}+(y M+\Delta)^{2}\right]}{\bar{y}^{2} D_{\phi B}^{2} D_{\Lambda B}^{4}},
$$

where $\bar{y}=1-y$, and we employed the shorthand notations [29]

$$
\begin{aligned}
D_{\phi B} & =-\frac{k_{\perp}^{2}+y M_{B}^{2}-y \bar{y} M^{2}+\bar{y} m_{\phi}^{2}}{\bar{y}}, \\
D_{\Lambda B} & =-\frac{k_{\perp}^{2}+y M_{B}^{2}-y \bar{y} M^{2}+\bar{y} \Lambda^{2}}{\bar{y}} .
\end{aligned}
$$

The $\delta$-function contributions are nonzero only at $y=0$, and arise from the local and nonlocal interactions. The local $\delta$-function term is given by

$$
\begin{aligned}
f_{\phi}^{(\delta)}(y)= & -\frac{\bar{\Lambda}^{8}}{\bar{M}^{2}} \int d k_{\perp}^{2} \int_{0}^{1} d z \frac{z^{3}}{\left(k_{\perp}^{2}+\Omega\right)^{4}} \delta(y) \\
= & \frac{1}{\bar{M}^{2}} \int d k_{\perp}^{2}\left[\log \frac{\Omega_{\phi}}{\Omega_{\Lambda}}\right. \\
& \left.+\frac{\bar{\Lambda}^{2}\left(11 \Omega_{\Lambda}^{2}-7 \Omega_{\Lambda} \Omega_{\phi}+2 \Omega_{\phi}^{2}\right)}{6 \Omega_{\Lambda}^{3}}\right] \delta(y),
\end{aligned}
$$

with 


$$
\Omega_{\phi}=k_{\perp}^{2}+m_{\phi}^{2}, \quad \Omega_{\Lambda}=k_{\perp}^{2}+\Lambda^{2} .
$$

The $\log \Omega_{\phi}$ term in Eq. (65) gives rise to the leading nonanalytic contribution, which is independent of the regularization method, as we have verified using various methods, including Pauli-Villars, dimensional regularization or a hadronic form factor. In the limit when $\Lambda \rightarrow \infty$, the second term in Eq. (65) $\sim \bar{\Lambda}^{2} / \Omega_{\Lambda}$ becomes a constant. Within dimensional regularization, the integral of a constant is defined to be zero, in which case the result coincides with that in Ref. [28],

$$
f_{\phi}^{(\delta)}(y) \underset{\Lambda \rightarrow \infty}{\rightarrow} \frac{1}{\bar{M}^{2}} \int d k_{\perp}^{2} \log \frac{\Omega_{\phi}}{\Omega_{\Lambda}} \delta(y) .
$$

The nonlocal $\delta$-function contribution, $\delta f_{B}^{(\delta)}$, in Eq. (62) is given by

$$
\begin{aligned}
\delta f_{\phi}^{(\delta)}(y)= & -\frac{\bar{\Lambda}^{8}}{\bar{M}^{2}} \int d k_{\perp}^{2} \int_{0}^{1} d z \frac{z^{4}}{\left(k_{\perp}^{2}+\Omega\right)^{4}} \delta(y) \\
= & \frac{1}{\bar{M}^{2}} \int d k_{\perp}^{2}\left[-4 \frac{\Omega_{\phi}}{\bar{\Lambda}^{2}} \log \frac{\Omega_{\phi}}{\Omega_{\Lambda}}\right. \\
& \left.-\frac{3 \Omega_{\Lambda}^{3}+13 \Omega_{\Lambda}^{2} \Omega_{\phi}-5 \Omega_{\Lambda} \Omega_{\phi}^{2}+\Omega_{\phi}^{3}}{3 \Omega_{\Lambda}^{3}}\right] \delta(y) .
\end{aligned}
$$

In the $\Lambda \rightarrow \infty$ limit the first term in the integrand of $\delta f_{\phi}^{(\delta)}$ vanishes, while the second term becomes a constant, independent of $k_{\perp}$. In dimensional regularization the latter can again be taken to be zero. The local function $f_{\phi}^{(\delta)}$, on the other hand, retains a dependence on $k_{\perp}$ through the $\log \Omega_{\phi}$ term, so that the splitting function for the rainbow diagram in Eq. (62) will reduce in this limit to the local splitting function. In the same limit, for the case $\phi=\pi$ and $B=N$, the integrand of Eq. (63) reduces to the familiar on-shell form found in the literature $[1,56,57]$,

$$
f_{\pi^{+} n}^{(\mathrm{on})}(y) \rightarrow \int d k_{\perp}^{2} \frac{y\left(k_{\perp}^{2}+y^{2} M^{2}\right)}{\left[k_{\perp}^{2}+y^{2} M^{2}+\bar{y} m_{\pi}^{2}\right]^{2}}
$$

for the specific dissociation $p \rightarrow \pi^{+} n$.

For the baryon coupling rainbow diagram, Fig. 1(b), the splitting function in Eq. (37) can be reduced to

$$
\begin{aligned}
f_{B \phi}^{(\mathrm{rbw})}(y)= & \frac{i C_{B \phi}^{2} \bar{\Lambda}^{8}}{f^{2}} \int \frac{d^{4} k}{(2 \pi)^{4}}\left[\frac{\bar{y} \bar{M}^{2}\left(\Delta^{2}-m_{\phi}^{2}\right)}{D_{B}^{2} D_{\phi} D_{\Lambda}^{4}}-\frac{\bar{y} \bar{M}^{2}}{D_{B}^{2} D_{\Lambda}^{4}}\right. \\
& \left.+\frac{(2-y) \bar{M} \Delta}{D_{B} D_{\phi} D_{\Lambda}^{4}}+\frac{1}{D_{\phi} D_{\Lambda}^{4}}\right] \delta\left(y-\frac{k^{+}}{p^{+}}\right) .
\end{aligned}
$$

Performing the $k^{-}$integral, this can then be expressed as a sum of on-shell, local and nonlocal off-shell, and $\delta$-function terms,

$$
\begin{aligned}
f_{B \phi}^{(\mathrm{rbw})}(y)= & \frac{C_{B \phi}^{2} \bar{M}^{2}}{(4 \pi f)^{2}}\left[f_{B}^{(\mathrm{on})}(y)+f_{B}^{(\mathrm{off})}(y)\right. \\
& \left.+4 \delta f_{B}^{(\mathrm{off})}(y)-f_{\phi}^{(\delta)}(y)\right]
\end{aligned}
$$

Note that the on-shell splitting functions for the baryon and meson couplings are equivalent, while the $\delta$-function contribution $f_{\phi}^{(\delta)}$ is as in Eq. (65). The off-shell contributions in Eq. (71) include local and nonlocal terms. The local off-shell contribution,

$$
f_{B}^{(\text {off })}(y)=\frac{2 \bar{\Lambda}^{8}}{\bar{M}} \int d k_{\perp}^{2} \frac{(y M+\Delta)}{\bar{y} D_{\phi B} D_{\Lambda B}^{4}},
$$

is similar to that derived in Refs. [25,28], while the nonlocal off-shell term is given by

$$
\delta f_{B}^{(\text {off })}(y)=\bar{\Lambda}^{8} \int d k_{\perp}^{2} \frac{y\left[k_{\perp}^{2}+(y M+\Delta)^{2}\right]}{\bar{y}^{2} D_{\phi B} D_{\Lambda B}^{5}} .
$$

In the $\Lambda \rightarrow \infty$ limit, the nonlocal term behaves as $\bar{\Lambda}^{8} / D_{\Lambda B}^{5} \sim 1 / \Lambda^{2}$, so vanishes, as expected.

For the Kroll-Ruderman diagram in Fig. 1(c), the splitting function in Eq. (38) for the dipole regulator becomes

$$
\begin{aligned}
f_{B}^{(\mathrm{KR})}(y)= & -\frac{2 i C_{B \phi}^{2} \bar{\Lambda}^{8}}{f^{2}} \int \frac{d^{4} k}{(2 \pi)^{4}}\left[\frac{(y M+\Delta) \bar{M}}{D_{\phi} D_{B} D_{\Lambda}^{4}}+\frac{1}{D_{\phi} D_{\Lambda}^{4}}\right] \\
& \times \delta\left(y-\frac{k^{+}}{p^{+}}\right),
\end{aligned}
$$

which after the $k^{-}$integration can be written in terms of the off-shell and $\delta$-function terms,

$$
f_{B}^{(\mathrm{KR})}(y)=\frac{C_{B \phi}^{2} \bar{M}^{2}}{(4 \pi f)^{2}}\left[-f_{B}^{(\mathrm{off})}(y)+2 f_{\phi}^{(\delta)}(y)\right],
$$

as given in Eqs. (65) and (72). (Note that the notation used here differs slightly from that of Ref. [29], where for strange octet baryons coupled to kaons the KrollRuderman function was labelled by $f_{Y K}^{(\mathrm{KR})}$; here we drop the meson label, as for a proton target the choice of baryon intermediate state uniquely specifies the meson, and we also label the $\delta$-function contribution by the baryon involved rather than the meson.) For the nonlocal gauge link contribution in Fig. 1(d), reduction of the Dirac matrices with the dipole form factor allows the corresponding splitting function $\delta f_{B}^{(\mathrm{KR})}$ to be rearranged as 


$$
\delta f_{B}^{(\mathrm{KR})}(y)=\frac{i C_{B \phi}^{2} \bar{\Lambda}^{8}}{f^{2}} \int \frac{d^{4} k}{(2 \pi)^{4}}\left[-\frac{4 y \bar{M}^{2}\left(\Delta^{2}-m_{\phi}^{2}\right)}{D_{\phi} D_{B} D_{\Lambda}^{5}}+\frac{4 y \bar{M}^{2}}{D_{B} D_{\Lambda}^{5}}-\frac{4 y(\bar{M} \Delta-2 p \cdot k)}{D_{\phi} D_{\Lambda}^{5}}\right] \delta\left(y-\frac{k^{+}}{p^{+}}\right) .
$$

After the $k^{-}$integration, this reduces to a sum of the nonlocal off-shell and $\delta$-function contributions,

$$
\delta f_{B}^{(\mathrm{KR})}(y)=\frac{C_{B \phi}^{2} \bar{M}^{2}}{(4 \pi f)^{2}}\left[-4 \delta f_{B}^{(\mathrm{off})}(y)-\delta f_{\phi}^{(\delta)}(y)\right],
$$

as given in Eqs. (68) and (73), respectively. From Eqs. (62), (71), (75) and (77) one can verify that the splitting functions satisfy the relation

$$
f_{\phi B}^{(\mathrm{rbw})}(y)=f_{B \phi}^{(\mathrm{rbw})}(y)+f_{B}^{(\mathrm{KR})}(y)+\delta f_{B}^{(\mathrm{KR})}(y),
$$

which generalizes the result in Ref. [28] to the nonlocal theory. Note that the local and nonlocal off-shell contributions $f_{B}^{(\text {off })}$ and $\delta f_{B}^{(\text {off })}$ cancel between the three terms on the right-hand side of Eq. (78). As noted above, in the $\Lambda \rightarrow \infty$ limit each of the functions induced by the nonlocal gauge link, $\delta f_{B}^{(\text {off })}$ and $\delta f_{\phi}^{(\delta)}$, vanishes, reproducing the local result from Ref. [25] that does not include the gauge link function $\delta f_{B}^{(\mathrm{KR})}$. Remarkably, the nonlocal generalization (78) means that gauge invariance is satisfied even in the presence of a finite form factor cutoff $\Lambda$.

A similar analysis can be applied to the tadpole and bubble diagrams in Fig. 1(e)-(g) in the presence of a hadronic form factor. From Eq. (43), the splitting function for the tadpole contribution with the dipole form factor can be written as

$$
f_{\phi}^{(\mathrm{tad})}(y)=-\frac{C_{\phi \phi^{\ddagger}} \bar{M}^{2}}{(4 \pi f)^{2}} f_{\phi}^{(\delta)}(y),
$$

where $f_{\phi}^{(\delta)}$ is given in Eq. (65). For the bubble diagram in Eq. (44) the corresponding splitting function is given by

$$
f_{\phi}^{(\mathrm{bub})}(y)=-\frac{C_{\phi \phi^{\dagger}} \bar{M}^{2}}{(4 \pi f)^{2}}\left[f_{\phi}^{(\delta)}(y)-\delta f_{\phi}^{(\delta)}(y)\right]
$$

where the nonlocal function $\delta f_{\phi}^{(\delta)}$ is given by Eq. (68). Finally, the splitting function for the nonlocal tadpole gauge link diagram in Fig. 1(g) from Eq. (47) with a dipole regulator is

$$
\delta f_{\phi}^{(\mathrm{tad})}(y)=\frac{C_{\phi \phi^{\dagger}} \bar{M}^{2}}{(4 \pi f)^{2}} \delta f_{\phi}^{(\delta)}(y) .
$$

Combining Eqs. (79)-(81), one finds that the tadpole and bubble diagrams satisfy the generalized relation

$$
f_{\phi}^{(\mathrm{bub})}(y)=f_{\phi}^{(\mathrm{tad})}(y)+\delta f_{\phi}^{(\mathrm{tad})}(y),
$$

which confirms the gauge invariance of the nonlocal theory.

\section{Decuplet splitting functions}

Turning now to the splitting functions for the decuplet baryon intermediate states in Fig. 1(h)-1(k), the contribution from the rainbow diagram with coupling to the pseudoscalar meson in Eq. (52) for the covariant dipole form factor (56) is given by

$$
\begin{aligned}
f_{\phi T}^{(\mathrm{rbw})}(y)= & \frac{i C_{T \phi}^{2} \bar{\Lambda}^{8}}{6 M_{T}^{2} f^{2}} \int \frac{d^{4} k}{(2 \pi)^{4}}\left[\frac{y\left(\bar{M}_{T}^{2}-m_{\phi}^{2}\right)^{2}\left(\Delta_{T}^{2}-m_{\phi}^{2}\right)}{D_{\phi}^{2} D_{T} D_{\Lambda}^{4}}-\frac{y\left(\bar{M}_{T}^{2}-m_{\phi}^{2}\right)\left(\bar{M}_{T}^{2}+2 \Delta_{T}^{2}-3 m_{\phi}^{2}\right)}{D_{\phi} D_{T} D_{\Lambda}^{4}}\right. \\
& +\frac{y\left(2 \bar{M}_{T}^{2}+\Delta_{T}^{2}-k^{2}-2 m_{\phi}^{2}\right)}{D_{T} D_{\Lambda}^{4}}+\frac{y}{D_{\phi}^{2} D_{\Lambda}^{4}}\left(4(p \cdot k)^{2}-2\left(\bar{M}_{T}^{2}-k^{2}\right) p \cdot k+\left(M_{T}^{2}-k^{2}\right)^{2}\right. \\
& \left.\left.+M\left(2 M_{T}^{3}-M^{3}-2 M^{2} M_{T}\right)-2 M k^{2}\left(2 M+M_{T}\right)\right)\right] \delta\left(y-\frac{k^{+}}{p^{+}}\right)
\end{aligned}
$$

where the coupling constants $C_{T \phi}$ for the decuplet intermediate states are listed in Table I, and the masses $\bar{M}_{T}$ and $\Delta_{T}$ here are defined in analogy with Eq. (58),

$$
\bar{M}_{T}=M+M_{T}, \quad \Delta_{T}=M_{T}-M
$$


After performing the $k^{-}$integration, the splitting function can be decomposed in terms of on-shell decuplet, end point, and local and nonlocal $\delta$-function terms,

$$
\begin{aligned}
f_{\phi T}^{(\mathrm{rbw})}(y)= & \frac{C_{T \phi}^{2} \bar{M}_{T}^{2}}{(4 \pi f)^{2}}\left[f_{T}^{(\mathrm{on})}(y)+f_{T}^{(\mathrm{on} \mathrm{end})}(y)-\frac{1}{18} f_{T}^{(\delta)}(y)\right. \\
& \left.+\frac{\bar{M}^{2}\left(\bar{M}_{T}^{2}-m_{\phi}^{2}\right)}{6 M_{T}^{2} \bar{M}_{T}^{2}}\left(f_{\phi}^{(\delta)}(y)-\delta f_{\phi}^{(\delta)}(y)\right)\right]
\end{aligned}
$$

As for the octet case, the first term in Eq. (85) is the on-shell splitting function for the meson rainbow with a decuplet spectator,

$$
\begin{aligned}
f_{T}^{(\mathrm{on})}(y)= & \frac{\bar{\Lambda}^{8}}{6 M_{T}^{2} \bar{M}_{T}^{2}} \int d k_{\perp}^{2} \frac{y\left(\bar{M}_{T}^{2}-m_{\phi}^{2}\right)}{\bar{y}} \\
& \times\left[\frac{\left(\bar{M}_{T}^{2}-m_{\phi}^{2}\right)\left(\Delta_{T}^{2}-m_{\phi}^{2}\right)}{D_{\phi T}^{2} D_{\Lambda T}^{4}}\right. \\
& \left.-\frac{3\left(\Delta_{T}^{2}-m_{\phi}^{2}\right)+4 M M_{T}}{D_{\phi T} D_{\Lambda T}^{4}}\right]
\end{aligned}
$$

where $D_{\phi T}$ and $D_{\Lambda T}$ are defined analogously to Eqs. (64)

$$
\begin{aligned}
& D_{\phi T}=-\frac{k_{\perp}^{2}+y M_{T}^{2}-y \bar{y} M^{2}+\bar{y} m_{\phi}^{2}}{\bar{y}}, \\
& D_{\Lambda T}=-\frac{k_{\perp}^{2}+y M_{T}^{2}-y \bar{y} M^{2}+\bar{y} \Lambda^{2}}{\bar{y}} .
\end{aligned}
$$

Since $\bar{\Lambda}^{8} / D_{\Lambda T}^{4} \rightarrow 1$ in the $\Lambda \rightarrow \infty$ limit, the decuplet onshell function (86) reduces to the pointlike result found in Ref. [27].

The function $f_{T}^{\text {(on end) }}$ in Eq. (85) is finite for finite values of $\Lambda$,

$$
\begin{aligned}
f_{T}^{(\text {on end })}(y)= & \frac{\bar{\Lambda}^{8}}{6 M_{T}^{2} \bar{M}_{T}^{2}} \int d k_{\perp}^{2} \frac{y}{\bar{y}^{2} D_{\Lambda T}^{4}} \\
& \times\left[k_{\perp}^{2}+y^{2} M^{2}-2 y\left(\bar{M}_{T}^{2}-M \Delta_{T}\right)\right. \\
& \left.-2 \bar{y} m_{\phi}^{2}+3 \bar{M}_{T}^{2}-4 M M_{T}\right]
\end{aligned}
$$

but in the $\Lambda \rightarrow \infty$ limit corresponds to the endpoint function in Ref. [27], with a singularity at $y=1$. To see this, first note that $D_{\Lambda T}$ in Eq. (87b) can be written in the form $\bar{y} D_{\Lambda T}=-\left(X_{T}+\bar{y} \Omega_{\Lambda}\right)$, where $X_{T}=y \Omega_{T}-y \bar{y} M^{2}$ and $\Omega_{T}=k_{\perp}^{2}+M_{T}^{2}$. In the $\Lambda \rightarrow \infty$ limit, one can then write the factor

$$
\begin{gathered}
\left.\frac{\bar{\Lambda}^{8}}{\bar{y}^{4} D_{\Lambda T}^{4}} \underset{\Lambda \rightarrow \infty}{\longrightarrow} \lim _{\Omega_{0} \rightarrow \infty} \int_{\Omega_{0}}^{\Omega_{T}} d t \frac{-4 y \bar{\Lambda}^{8}}{\left(y t-y \bar{y} M^{2}+\bar{y} \Omega_{\Lambda}\right)^{5}}\right|_{\Lambda \rightarrow \infty} \\
=\frac{\bar{\Lambda}^{6}}{\bar{y}^{3} \Omega_{\Lambda}^{3}} \lim _{\Omega_{0} \rightarrow \infty}\left(\frac{\bar{\Lambda}^{2} \bar{y}^{3} \Omega_{\Lambda}^{3}}{\bar{y}^{4} D_{\Lambda T}^{4}}-\frac{\bar{\Lambda}^{2} \bar{y}^{3} \Omega_{\Lambda}^{3}}{\bar{y}^{4} D_{0}^{4}}\right)_{\Lambda \rightarrow \infty},
\end{gathered}
$$

where $\bar{y} D_{0}=-\left(X_{0}+\bar{y} \Omega_{\Lambda}\right)$, with $X_{0}=y \Omega_{0}-y \bar{y} M^{2}$ and $\Omega_{0}$ is a $\Lambda$-independent constant. At finite $\Lambda$, the term involving $D_{0}$ vanishes; however, care must be taken when evaluating this for $\Lambda \rightarrow \infty$. Replacing $\bar{y} \Omega_{\Lambda}$ in the first and second terms in Eq. (89) by $\left(-\bar{y} D_{\Lambda T}-X_{T}\right)$ and $\left(-\bar{y} D_{0}-X_{0}\right)$, respectively, one obtains

$$
\begin{aligned}
\frac{\bar{\Lambda}^{8}}{\bar{y}^{4} D_{\Lambda T}^{4}} \underset{\Lambda \rightarrow \infty}{\longrightarrow}-\frac{\bar{\Lambda}^{6}}{\bar{y}^{3} \Omega_{\Lambda}^{3} \Omega_{0} \rightarrow \infty}\left[\left(\frac{\bar{\Lambda}^{2}}{\bar{y} D_{\Lambda T}}-\frac{\bar{\Lambda}^{2}}{\bar{y} D_{0}}\right)\right. \\
+3\left(\frac{\bar{\Lambda}^{2} X_{T}}{\bar{y}^{2} D_{\Lambda T}^{2}}-\frac{\bar{\Lambda}^{2} X_{0}}{\bar{y}^{2} D_{0}^{2}}\right)+3\left(\frac{\bar{\Lambda}^{2} X_{T}^{2}}{\bar{y}^{3} D_{\Lambda T}^{3}}-\frac{\bar{\Lambda}^{2} X_{0}^{2}}{\bar{y}^{3} D_{0}^{3}}\right) \\
\left.+\left(\frac{\bar{\Lambda}^{2} X_{T}^{3}}{\bar{y}^{4} D_{\Lambda T}^{4}}-\frac{\bar{\Lambda}^{2} X_{0}^{3}}{\bar{y}^{4} D_{0}^{4}}\right)\right]_{\Lambda \rightarrow \infty} .
\end{aligned}
$$

Since in the $\Lambda \rightarrow \infty$ limit one has $\bar{y} D_{\Lambda T} \rightarrow$ $-\bar{\Lambda}^{2}\left(\bar{y}+X_{T} / \Omega_{\Lambda}\right)$, the first term in parentheses in Eq. (90) can be written

$$
\left(\frac{\bar{\Lambda}^{2}}{\bar{y} D_{\Lambda T}}-\frac{\bar{\Lambda}^{2}}{\bar{y} D_{0}}\right)_{\Lambda \rightarrow \infty}=-\left(\frac{1}{\bar{y}+X_{T} / \Omega_{\Lambda}}-\frac{1}{\bar{y}+X_{0} / \Omega_{\Lambda}}\right)_{\Lambda \rightarrow \infty},
$$

where we have taken $\Omega_{0} \ll \Lambda^{2}$. The right-hand side of Eq. (91) has the properties that it vanishes if $\bar{y} \neq 0$, is divergent if $\bar{y}=0$, and becomes $\log \left(X_{T} / X_{0}\right)$ when integrated over $\bar{y}$, so that it can be represented by a $\delta$ function,

$$
\left(\frac{\bar{\Lambda}^{2}}{\bar{y} D_{\Lambda T}}-\frac{\bar{\Lambda}^{2}}{\bar{y} D_{0}}\right)_{\Lambda \rightarrow \infty}=\delta(\bar{y}) \log \frac{X_{T}}{X_{0}} .
$$

Similarly, for the $1 /\left(\bar{y} D_{\Lambda T}\right)^{n}$ terms in Eq. (89) with $n \geq 2$, one can write in the $\Lambda \rightarrow \infty$ limit

$$
\left.\frac{\bar{\Lambda}^{2} X_{T}^{n-1}}{(-\bar{y})^{n} D_{\Lambda T}^{n}}\right|_{\Lambda \rightarrow \infty}=\left.\frac{\left(X_{T} / \Lambda^{2}\right)^{n-1}}{\left(\bar{y}+X_{T} / \Omega_{\Lambda}\right)^{n}}\right|_{\Lambda \rightarrow \infty}=\frac{\delta(\bar{y})}{n-1}, \quad n \geq 2 .
$$

Since the same result is obtained when $X_{T}$ is replaced by $X_{0}$, the $1 /\left(\bar{y} D_{\Lambda T}\right)^{n}$ and $1 /\left(\bar{y} D_{0}\right)^{n}$ terms cancel for $n \geq 2$, and one obtains

$$
\frac{\bar{\Lambda}^{8}}{\bar{y}^{4} D_{\Lambda T}^{4}} \underset{\Lambda \rightarrow \infty}{\longrightarrow}-\frac{1}{\bar{y}^{3}} \log \frac{\Omega_{T}}{\Omega_{0}} \delta(\bar{y})=-\frac{1}{\bar{y}^{3}}\left(\log \frac{\Omega_{T}}{\mu^{2}}-1\right) \delta(\bar{y}),
$$


where $\mu$ is defined such that $\log \left(\Omega_{T} / \mu^{2}\right)=\log \left(\Omega_{T} / \Omega_{0}\right)+1$. With this result, one can finally write the endpoint splitting function in the $\Lambda \rightarrow \infty$ limit as

$$
f_{T}^{(\text {on end })}(y) \underset{\Lambda \rightarrow \infty}{\longrightarrow} \frac{1}{6 M_{T}^{2} \bar{M}_{T}^{2}} \int d k_{\perp}^{2}\left\{\left[\Omega_{T}-2\left(\Delta_{T}^{2}-m_{\phi}^{2}\right)-6 M M_{T}\right] \log \frac{\Omega_{T}}{\mu^{2}}-\Omega_{T}+2\left(\Delta_{T}^{2}-m_{\phi}^{2}\right)+6 M M_{T}\right\} \delta(\bar{y}) .
$$

This expression is identical to that for the endpoint term in Ref. [27], except for the $k_{\perp}$-independent terms in (95). For dimensional regularization, however, these are again defined to be zero, so that the result does indeed match that in [27].

Note also that at finite values of $\Lambda$ the sum of the on-shell function $f_{T}^{(\text {on })}$ in Eq. (86) and the on-shell endpoint function $f_{T}^{\text {(on end) }}$ in Eq. (88) gives the usual result found in the literature by taking the pole contribution alone $[1,2,27,54,57]$,

$$
f_{T}^{\text {(on })}(y)+f_{T}^{\text {(on end })}(y)=\frac{\bar{\Lambda}^{8}}{6 M_{T}^{2} \bar{M}_{T}^{2}} \int d k_{\perp}^{2} \frac{y\left[k_{\perp}^{2}+\left(\Delta_{T}+y M\right)^{2}\right]\left[k_{\perp}^{2}+\left(\bar{M}_{T}-y M\right)^{2}\right]^{2}}{\bar{y}^{4} D_{\phi T}^{2} D_{\Lambda T}^{4}},
$$

for $0<y<1$. Separately, however, the on-shell and endpoint functions are not guaranteed to be positive definite for large values of $m_{\phi}$, since the individual functions do not correspond to physical processes [58]. The combined contribution in Eq. (96) is, however, positive for any combination of masses and kinematics.

For the $\delta$-function contributions at $y=0$, there are three distinct terms in the decuplet rainbow function $f_{\phi T}^{(\mathrm{rbw})}$. The new decuplet $\delta$-function term in Eq. (85) for the nonlocal case is given by

$$
\begin{aligned}
f_{T}^{(\delta)}(y) & =\frac{\bar{\Lambda}^{8}}{M_{T}^{2} \bar{M}_{T}^{2}} \int d k_{\perp}^{2} \int_{0}^{1} d z \frac{z^{3}}{\left(k_{\perp}^{2}+\Omega\right)^{3}} \delta(y) \\
& =\frac{1}{M_{T}^{2} \bar{M}_{T}^{2}} \int d k_{\perp}^{2} \frac{1}{2 \Omega_{\Lambda}^{2}}\left[6 \Omega_{\Lambda}^{2} \Omega_{\phi} \log \frac{\Omega_{\phi}}{\Omega_{\Lambda}}+\left(\Omega_{\phi}-\Omega_{\Lambda}\right)\left(\Omega_{\phi}^{2}-5 \Omega_{\phi} \Omega_{\Lambda}-2 \Omega_{\Lambda}^{2}\right)\right] \delta(y),
\end{aligned}
$$

where $\Omega_{\phi}$ and $\Omega_{\Lambda}$ are as in Eq. (66). In the $\Lambda \rightarrow \infty$ limit, only the first term in the integrand of Eq. (97) survives, so that the local limit of the function $f_{T}^{(\delta)}$ is

$$
f_{T}^{(\delta)}(y) \underset{\Lambda \rightarrow \infty}{\longrightarrow} \frac{3}{M_{T}^{2} \bar{M}_{T}^{2}} \int d k_{\perp}^{2}\left[\Omega_{\phi} \log \frac{\Omega_{\phi}}{\mu^{2}}-\Omega_{\phi}\right] \delta(y),
$$

where the constant $\mu$ here is defined by $\log \left(\Omega_{\phi} / \mu^{2}\right)=\log \left(\Omega_{\phi} / \Omega_{\Lambda}\right)+17 / 6$.

The remaining $\delta$-function terms in Eq. (85), namely, the local $f_{\phi}^{(\delta)}$ and nonlocal $\delta f_{\phi}^{(\delta)}$ functions, are given in Eqs. (65) and (68), respectively. The combined contribution of the $\delta$-function terms to $f_{\phi T}^{(\mathrm{rbw})}$ in the local limit is then

$$
\frac{1}{18}\left[\frac{3 \bar{M}^{2}\left(\bar{M}_{T}^{2}-m_{\phi}^{2}\right)}{M_{T}^{2} \bar{M}_{T}^{2}} f_{\phi}^{(\delta)}-f_{T}^{(\delta)}\right] \underset{\Lambda \rightarrow \infty}{\longrightarrow} \frac{1}{6 M_{T}^{2} \bar{M}_{T}^{2}} \int d k_{\perp}^{2}\left[\Omega_{\phi}+\left(\bar{M}_{T}^{2}-m_{\phi}^{2}-\Omega_{\phi}\right) \log \frac{\Omega_{\phi}}{\mu^{2}}\right] \delta(y) .
$$

Note that this expression differs from the total local $\delta(y)$ contribution in Ref. [27], which was computed using the projector $P_{\alpha \beta}$ in Eq. (50) but with $Z=-1 / 2$ for the interaction $\Theta^{\mu \nu}$ in Eq. (2). As discussed in Ref. [27], for values of the off-shell parameter $Z \neq-1 / 2$, the additional interaction term $\sim \gamma_{\mu} \gamma_{\nu}$ in $\Theta^{\mu \nu}$ contributes only to the $\delta(y)$ contribution. The result here supercedes that in Ref. [27]. 
For the decuplet baryon coupling rainbow diagram in Fig. 1(i), reduction of the $\gamma$-matrices in Eq. (53) yields

$$
\begin{aligned}
f_{T \phi}^{(\mathrm{rbw})}(y)= & \frac{i C_{T \phi}^{2} \bar{\Lambda}^{8}}{6 M_{T}^{2} f^{2}} \int \frac{d^{4} k}{(2 \pi)^{4}}\left[\frac{\bar{y}\left(\bar{M}_{T}^{2}-m_{\phi}^{2}\right)^{2}\left(\Delta_{T}^{2}-m_{\phi}^{2}\right)}{D_{\phi} D_{T}^{2} D_{\Lambda}^{4}}+\frac{\bar{y}\left[\left(k^{2}+m_{\phi}^{2}\right)\left(2 \bar{M}_{T}^{2}+\Delta_{T}^{2}-m_{\phi}^{2}\right)-\bar{M}_{T}^{4}-2 \bar{M}_{T}^{2} \Delta_{T}^{2}-k^{4}\right]}{D_{T}^{2} D_{\Lambda}^{4}}\right. \\
& -\frac{\left(\bar{M}_{T}^{2}-m_{\phi}^{2}\right)\left[(y-2) M_{T}^{2}-2 y M M_{T}+(y+2)\left(M^{2}-m_{\phi}^{2}\right)\right]}{D_{T} D_{\phi} D_{\Lambda}^{4}}+\frac{(y+2)\left(2 M^{2}-m_{\phi}^{2}-k^{2}\right)+\left(y M_{T}+2 M\right) 2 M_{T}}{D_{T} D_{\Lambda}^{4}} \\
& \left.+\frac{\bar{M}_{T}^{2}+2 y \bar{M}_{T} M-2 y p \cdot k+3 k^{2}}{D_{\phi} D_{\Lambda}^{4}}\right] \delta\left(y-\frac{k^{+}}{p^{+}}\right)
\end{aligned}
$$

Integrating over $k^{-}$, the splitting function for the decuplet coupling rainbow diagram can be written analogously to the function $f_{\phi T}^{(\mathrm{rbw})}$ in Eq. (85),

$$
\begin{aligned}
f_{T \phi}^{\text {(rbw) }}(y)= & \frac{C_{T \phi}^{2} \bar{M}_{T}^{2}}{(4 \pi f)^{2}}\left[f_{T}^{(\text {on })}(y)+f_{T}^{(\text {on end })}(y)-2\left(f_{T}^{(\text {off })}(y)+f_{T}^{(\text {off end })}(y)-2 \delta f_{T}^{(\text {off })}(y)\right)\right. \\
& \left.+\frac{1}{18}\left(f_{T}^{(\delta)}(y)-3 \delta f_{T}^{(\delta)}(y)\right)-\frac{\bar{M}^{2}\left(\bar{M}_{T}^{2}+3 m_{\phi}^{2}\right)}{6 M_{T}^{2} \bar{M}_{T}^{2}} f_{\phi}^{(\delta)}(y)\right]
\end{aligned}
$$

The first term in Eq. (101) is the on-shell splitting function for the decuplet baryon rainbow, and is identical to that for the meson coupling rainbow in Eq. (85). The second term is the same as the endpoint function contribution in Eq. (88).

The off-shell decuplet contributions to $f_{T \phi}^{\text {(rbw) }}$ appear as three individual terms-a local off-shell piece, $f_{T}^{(\text {on })}$, an off-shell endpoint contribution, $f_{T}^{(\text {off end) }}$, and a purely nonlocal term, $\delta f_{T}^{(\text {off })}$. The local off-shell function is given by

$$
f_{T}^{(\text {off })}(y)=\frac{\bar{\Lambda}^{8}}{6 M_{T}^{2} \bar{M}_{T}^{2}} \int d k_{\perp}^{2} \frac{\left(\bar{M}_{T}^{2}-m_{\phi}^{2}\right)\left[\bar{y}\left(M^{2}-m_{\phi}^{2}\right)-(1+y) M_{T}^{2}\right]}{\bar{y} D_{\phi T} D_{\Lambda T}^{4}},
$$

which in the $\Lambda \rightarrow \infty$ limit reduces to

$$
f_{T}^{(\text {off })}(y) \underset{\Lambda \rightarrow \infty}{\rightarrow} \frac{1}{6 M_{T}^{2} \bar{M}_{T}^{2}} \int d k_{\perp}^{2} \frac{\left(\bar{M}_{T}^{2}-m_{\phi}^{2}\right)\left[\bar{y}\left(M^{2}-m_{\phi}^{2}\right)-(1+y) M_{T}^{2}\right]}{\bar{y} D_{\phi T}} .
$$

In addition to the endpoint function for the on-shell contribution in Eq. (88), a separate endpoint contribution exists for the off-shell case, $f_{T}^{\text {(off end) }}$, and is given by

$$
f_{T}^{\text {(off end) }}(y)=-\frac{\bar{\Lambda}^{8}}{6 M_{T}^{2} \bar{M}_{T}^{2}} \int d k_{\perp}^{2} \frac{\left[k_{\perp}^{2}+\bar{y}^{2} M^{2}+\bar{y}\left(\bar{M}_{T}^{2}-m_{\phi}^{2}\right)-M_{T}^{2}\right]}{\bar{y} D_{\Lambda T}^{4}} .
$$

Using the relation in Eq. (94), one can show that in the $\Lambda \rightarrow \infty$ limit this term is proportional to a $\delta$ function at $y=1$,

$$
f_{T}^{\text {(off end) }}(y) \underset{\Lambda \rightarrow \infty}{\rightarrow} \frac{1}{6 M_{T}^{2} \bar{M}_{T}^{2}} \int d k_{\perp}^{2}\left\{\left[\Omega_{T}-2 M_{T}^{2}\right] \log \frac{\Omega_{T}}{\mu^{2}}-\Omega_{T}\right\} \delta(\bar{y}) .
$$

As for the octet case in Eq. (73), the decuplet splitting function also includes a nonlocal decuplet off-shell term, given by

$$
\delta f_{T}^{(\text {off })}(y)=\frac{\bar{\Lambda}^{8}}{6 M_{T}^{2} \bar{M}_{T}^{2}} \int d k_{\perp}^{2} \frac{y\left[k_{\perp}^{2}+\left(y M-\overline{M_{T}}\right)^{2}\right]^{2}\left[k_{\perp}^{2}+\left(y M+\Delta_{T}\right)^{2}\right]}{\bar{y}^{4} D_{\phi T} D_{\Lambda T}^{5}},
$$

The presence of the $1 / D_{\Lambda T}^{5}$ in the integrand of (106) ensures that in the $\Lambda \rightarrow \infty$ limit the nonlocal function vanishes, $\delta f_{T}^{(\text {off })} \rightarrow 0$. 
For the $\delta$-function contributions at $y=0$, the local terms $f_{\phi}^{(\delta)}$ and $f_{T}^{(\delta)}$ in Eq. (101) are given above in Eqs. (65) and (68), respectively, while the new nonlocal $\delta$-function term, $\delta f_{T}^{(\delta)}$, is given by

$$
\delta f_{T}^{(\delta)}(y)=\frac{\bar{\Lambda}^{8}}{M_{T}^{2} \bar{M}_{T}^{2}} \int d k_{\perp}^{2} \frac{1}{\Omega_{\Lambda}^{3}} \delta(y)
$$

As with the other nonlocal contributions, this term also vanishes in the $\Lambda \rightarrow \infty$ limit.

The final diagram in Fig. 1 is that for the Kroll-Ruderman contribution with a decuplet intermediate state, Fig. 1(j). The splitting function corresponding to this diagram, after reducing the $\gamma$-matrices in Eq. (54), can be written

$$
\begin{aligned}
f_{T}^{(\mathrm{KR})}(y)= & -i \frac{C_{T \phi}^{2} \bar{\Lambda}^{8}}{3 M_{T}^{2} f^{2}} \int \frac{d^{4} k}{(2 \pi)^{4}}\left[\frac{\left(\bar{M}_{T}^{2}-m_{\phi}^{2}\right)\left[(1+y) M_{T}^{2}-\bar{y}\left(M^{2}-m_{\phi}^{2}\right)\right]}{D_{\phi} D_{T} D_{\Lambda}^{4}}\right. \\
& \left.+\frac{(1-y) k^{2}-2(1+y) p \cdot k+y\left(2 M^{2}+\bar{M}_{T}^{2}\right)+\bar{M}_{T}^{2}}{D_{\phi} D_{\Lambda}^{4}}-\frac{2 y M_{T}^{2}+\bar{y}\left(k^{2}+m_{\phi}^{2}-2 M \bar{M}_{T}\right)}{D_{T} D_{\Lambda}^{4}}\right] \delta\left(y-\frac{k^{+}}{p^{+}}\right) .
\end{aligned}
$$

After integrating over $k^{-}$, the splitting function for the decuplet KR diagram can be expressed in terms of local and nonlocal off-shell and $\delta$-function terms,

$$
f_{T}^{(\mathrm{KR})}(y)=\frac{C_{T \phi}^{2} \bar{M}_{T}^{2}}{(4 \pi f)^{2}}\left[2\left(f_{T}^{(\text {off })}(y)+f_{T}^{(\text {off end })}(y)\right)-\frac{1}{9}\left(f_{T}^{(\delta)}(y)-\delta f_{T}^{(\delta)}(y)\right)+\frac{\bar{M}^{2}\left(\bar{M}_{T}^{2}+m_{\phi}^{2}\right)}{3 M_{T}^{2} \bar{M}_{T}^{2}} f_{\phi}^{(\delta)}(y)\right],
$$

each of which has been defined previously. Finally, the splitting function for the additional decuplet diagram induced by the gauge link, Fig. 1(k), is obtained from Eq. (55),

$$
\begin{aligned}
\delta f_{T}^{(\mathrm{KR})}(y)= & -2 i \frac{C_{T \phi}^{2} \bar{\Lambda}^{8}}{3 M_{T}^{2} f^{2}} \int \frac{d^{4} k}{(2 \pi)^{4}} y\left[\frac{\left(\bar{M}_{T}^{2}-m_{\phi}^{2}\right)^{2}\left(\Delta_{T}^{2}-m_{\phi}^{2}\right)}{D_{\phi} D_{T} D_{\Lambda}^{5}}+\frac{\left(k^{2}+m_{\phi}^{2}\right)\left(2 \bar{M}_{T}^{2}+\Delta_{T}^{2}-m_{\phi}^{2}\right)-\left(\bar{M}_{T}^{2}-2 \Delta_{T}^{2}\right) \bar{M}_{T}^{2}-k^{4}}{D_{T} D_{\Lambda}^{5}}\right. \\
& +\frac{1}{D_{\phi} D_{\Lambda}^{5}}\left(4(p \cdot k)^{2}+3 m_{\phi}^{4}-\left(3 k^{2}+3 \bar{M}_{T}^{2}+\Delta_{T}^{2}-\bar{M}_{T} \Delta_{T}\right) m_{\phi}^{2}-\left(4 k^{2}-6 m_{\phi}^{2}+2 \bar{M}_{T}^{2}\right)(p \cdot k)\right. \\
& \left.\left.+k^{2} \bar{M}_{T}^{2}+\bar{M}_{T}^{3} \Delta_{T}+k^{4}\right)-\frac{1}{D_{\Lambda}^{5}}\left(3 M_{T}^{2}+5 M^{2}+4 M M_{T}-3 m_{\phi}^{2}-6 p \cdot k\right)\right] \delta\left(y-\frac{k^{+}}{p^{+}}\right) .
\end{aligned}
$$

With integration over $k^{-}$, the splitting function for the nonlocal KR gauge link diagram can be simplified to a sum of nonlocal off-shell and $\delta$-function contributions,

$$
\delta f_{T}^{(\mathrm{KR})}(y)=\frac{C_{T \phi}^{2} \bar{M}_{T}^{2}}{(4 \pi f)^{2}}\left[-4 \delta f_{T}^{(\mathrm{off})}(y)+\frac{1}{18} \delta f_{T}^{(\delta)}(y)-\frac{\bar{M}^{2}\left(\bar{M}_{T}^{2}-m_{\phi}^{2}\right)}{6 M_{T}^{2} \bar{M}_{T}^{2}} \delta f_{\phi}^{(\delta)}(y)\right] .
$$

From Eqs. (85), (100), (109) and (111), one can then explicitly verify that gauge invariance for the decuplet baryon contributions is satisfied through the relation

$$
f_{\phi T}^{(\mathrm{rbw})}(y)=f_{T \phi}^{(\mathrm{rbw})}(y)+f_{T}^{(\mathrm{KR})}(y)+\delta f_{T}^{(\mathrm{KR})}(y) .
$$

This generalizes the result from Ref. [29] to nonlocal interactions in the presence of vertex functions parametrizing the extended nature of the proton.

\section{Leading nonanalytic behavior}

Having derived the complete set of splitting functions for the one-loop diagrams in Fig. 1 for the dissociation of a proton to a pseudoscalar meson $(\phi)$ and an SU(3) octet $(B)$ or decuplet $(T)$ baryon, in the rest of this section we discuss the characteristics of each of the functions and illustrate their relative shapes and magnitudes numerically. The full set of functions includes 8 basis functions that are nonzero in the local limit, $\left\{f_{B}^{\text {(on })}, f_{B}^{(\text {off })}, f_{T}^{\text {(on })}, f_{T}^{\text {(on end })}\right.$, $\left.f_{T}^{\text {(off) }}, f_{T}^{\text {(off end })}, f_{T}^{(\delta)}, f_{\phi}^{(\delta)}\right\}$, and 4 nonlocal functions, 
$\left\{\delta f_{B}^{(\text {off })}, \delta f_{T}^{\text {(off) }}, \delta f_{T}^{(\delta)}, \delta f_{\phi}^{(\delta)}\right\}$, that vanish for pointlike particles. All of the diagrams in Fig. 1 are then represented by splitting functions that can be written as linear combinations of these basis functions.

Before presenting the numerical results for the splitting functions for the case of the covariant dipole form factor in Eq. (56), we first identify some features of the basis functions that do not depend on details of the regularization method, but are entirely determined by the infrared behavior of the chiral loops. Namely, expanding the lowest moments $\left\langle f_{i}\right\rangle$ of the basis splitting functions,

$$
\left\langle f_{i}\right\rangle=\int_{0}^{1} d y f_{i}(y)
$$

as a series in the pseudoscalar meson mass $m_{\phi}$, the coefficients of terms that are nonanalytic (NA) in $m_{\phi}^{2}$ (either odd powers of $m_{\phi}$ or logarithms of $m_{\phi}$ ) are determined by the low-energy properties of the nucleon and do not depend on the ultraviolet behavior of the functions [19-23]. In particular, the moments of the on-shell and off-shell functions $f_{B}^{(\text {on })}, f_{B}^{\text {(off) }}, f_{T}^{\text {(on) }}, f_{T}^{\text {(off) }}$ and the $\delta$-function terms $f_{\phi}^{(\delta)}$ and $f_{T}^{(\delta)}$ all receive NA contributions, while the purely nonlocal functions and the decuplet endpoint contributions $f_{T}^{\text {(on end) }}$ and $f_{T}^{\text {(off end) }}$ are entirely analytic.

For the octet intermediate states, we find the NA contribution to the on-shell moment $\left\langle f_{B}^{(\text {on })}\right\rangle$ is given by

$$
\left.\bar{M}^{2}\left\langle f_{B}^{\text {(on })}\right\rangle\right|_{\mathrm{NA}}= \begin{cases}\left(4 m_{\phi}^{2}-6 \Delta^{2}\right) \log m_{\phi}^{2}+6 R \Delta \log \frac{\Delta-R}{\Delta+R}, & \Delta>m_{\phi}, \\ \left(4 m_{\phi}^{2}-6 \Delta^{2}\right) \log m_{\phi}^{2}+6 \bar{R} \Delta\left(\pi-2 \arctan \frac{\Delta}{\bar{R}}\right), & \Delta<m_{\phi},\end{cases}
$$

where $R=\sqrt{\Delta^{2}-m_{\phi}^{2}}$ and $\bar{R}=\sqrt{m_{\phi}^{2}-\Delta^{2}}$. This agrees with the result found in Ref. [29] for strange octet contributions. In particular, for the latter case, when $\Delta<m_{\phi}$, the mass difference $\Delta$ approaches zero first in the chiral limit, $m_{\phi} \rightarrow 0$. The resulting LNA term is then simply $4 m_{\phi}^{2} \log m_{\phi}^{2}$, consistent with Refs. [20-24,27]. For the case $\Delta>m_{\phi}$, expanding $R$ as $R=\Delta-m_{\phi}^{2} / 2 \Delta+$ $\mathcal{O}\left(m_{\phi}^{4}\right)$ one finds that the $\Delta^{2} \log m_{\phi}^{2}$ terms cancel, leaving behind the same LNA behavior $\sim m_{\phi}^{2} \log m_{\phi}^{2}$,

$$
\begin{aligned}
\left.\bar{M}^{2}\left\langle f_{B}^{(\text {on })}\right\rangle\right|_{\text {LNA }}= & \left(4 m_{\phi}^{2}-6 \Delta^{2}\right) \log m_{\phi}^{2}+6 \Delta^{2} \log m_{\phi}^{2} \\
& -3 m_{\phi}^{2} \log m_{\phi}^{2} \\
= & m_{\phi}^{2} \log m_{\phi}^{2}, \quad \Delta>m_{\phi}
\end{aligned}
$$

but with a coefficient that is now 4 times smaller than for the $\Delta<m_{\phi}$ case.

For the off-shell moment $\left\langle f_{B}^{(\text {off })}\right\rangle$, the NA contribution is

$$
\left.\bar{M}^{2}\left\langle f_{B}^{(\text {off })}\right\rangle\right|_{\mathrm{NA}}= \begin{cases}-2 m_{\phi}^{2} \log m_{\phi}^{2}-\frac{2 R^{3}}{M_{B}} \log \frac{\Delta-R}{\Delta+R}, & \Delta>m_{\phi}, \\ -2 m_{\phi}^{2} \log m_{\phi}^{2}+\frac{2 \bar{R}^{3}}{M_{B}}\left(\pi-2 \arctan \frac{\Delta}{\bar{R}}\right), & \Delta<m_{\phi} .\end{cases}
$$

The LNA behavior of the moment, $\left\langle f_{\phi}^{(\delta)}\right\rangle$, of the $\delta$-function term is

$$
\left.\bar{M}^{2}\left\langle f_{\phi}^{(\delta)}\right\rangle\right|_{\mathrm{LNA}}=-m_{\phi}^{2} \log m_{\phi}^{2}
$$

These results generalize the LNA expressions given for hyperons and kaons in Ref. [29].

For the decuplet intermediate states, the NA term for the on-shell moment $\left\langle f_{T}^{(\text {on })}\right\rangle$ is

$$
\left.\bar{M}_{T}^{2}\left\langle f_{T}^{(\mathrm{on})}\right\rangle\right|_{\mathrm{NA}}= \begin{cases}\frac{\left(8 m_{\phi}^{2}-12 \Delta_{T}^{2}\right)}{3} \log m_{\phi}^{2}+4 R_{T} \Delta_{T} \log \frac{\Delta_{T}-R_{T}}{\Delta_{T}+R_{T}}, & \Delta_{T}>m_{\phi}, \\ \frac{\left(8 m_{\phi}^{2}-12 \Delta_{T}^{2}\right)}{3} \log m_{\phi}^{2}+4 \bar{R}_{T} \Delta_{T}\left(\pi-2 \arctan \frac{\Delta_{T}}{\bar{R}_{T}}\right), & \Delta_{T}<m_{\phi},\end{cases}
$$


where $R_{T}=\sqrt{\Delta_{T}^{2}-m_{\phi}^{2}}$ and $\bar{R}_{T}=\sqrt{m_{\phi}^{2}-\Delta_{T}^{2}}$. For the case $\Delta_{T}<m_{\phi}$, one finds in the $\Delta_{T} \rightarrow 0$ limit the LNA behavior $\frac{8}{3} m_{\phi}^{2} \log m_{\phi}^{2}$. For $\Delta_{T}>m_{\phi}$, one may again expand $R_{T}$ as $R_{T}=\Delta_{T}-m_{\phi}^{2} / 2 \Delta_{T}+\mathcal{O}\left(m_{\phi}^{4}\right)$, and note that the LNA term remains $\sim m_{\phi}^{2} \log m_{\phi}^{2}$ due to a cancellation of the terms proportional to $\Delta_{T}^{2} \log m_{\phi}^{2}$,

$$
\begin{aligned}
\left.\bar{M}_{T}^{2}\left\langle f_{T}^{(\mathrm{on})}\right\rangle\right|_{\mathrm{LNA}} & =\frac{\left(8 m_{\phi}^{2}-12 \Delta_{T}^{2}\right)}{3} \log m_{\phi}^{2}+4 \Delta_{T}^{2} \log m_{\phi}^{2}-2 m_{\phi}^{2} \log m_{\phi}^{2} \\
& =\frac{2}{3} m_{\phi}^{2} \log m_{\phi}^{2}, \quad \Delta_{T}>m_{\phi} .
\end{aligned}
$$

In both cases, therefore, the LNA term is given by $m_{\phi}^{2} \log m_{\phi}^{2}$, although the coefficient for $\Delta_{T}>m_{\phi}$ is 4 times smaller than that for $\Delta_{T}<m_{\phi}$ in the chiral limit.

The NA contribution to the moment of the decuplet off-shell function $\left\langle f_{T}^{(\text {off })}\right\rangle$ is given by

$$
\left.\bar{M}_{T}^{2}\left\langle f_{T}^{\text {(off })}\right\rangle\right|_{\mathrm{NA}}= \begin{cases}\frac{2}{3} m_{\phi}^{2} \log m_{\phi}^{2}+\frac{4 R_{T}^{3}}{3 M_{T}} \log \frac{\Delta_{T}-R_{T}}{\Delta_{T}+R_{T}}, & \Delta_{T}>m_{\phi}, \\ \frac{2}{3} m_{\phi}^{2} \log m_{\phi}^{2}-\frac{4 \bar{R}_{T}^{3}}{3 M_{T}}\left(\pi-2 \arctan \frac{\Delta_{T}}{\bar{R}_{T}}\right), & \Delta_{T}<m_{\phi} .\end{cases}
$$

The decuplet $\delta$-function moment does not have an LNA term, but has contributions at higher order in $m_{\pi}$,

$$
\left.\bar{M}_{T}^{2}\left\langle f_{T}^{(\delta)}\right\rangle\right|_{\mathrm{LNA}}=0
$$

The decuplet results for the total LNA behavior coincide with those for the $\pi \Delta$ intermediate states in Ref. [27], arising from the $f_{T}^{(\text {on })}$ and $f_{\phi}^{(\delta)}$ terms in Eq. (85), if the $\pi N \Delta$ coupling constant $g_{\pi N \Delta}$ in [27] is related to the meson-octet-decuplet coupling constant $\mathcal{C}$ in Eq. (1) by $g_{\pi N \Delta}^{2}=\mathcal{C}^{2} /\left(2 f^{2}\right)$.

We stress that these results are completely general, depending only on the infrared properties of pseudoscalar meson loops, following directly from the symmetries of the chiral Lagrangian. They are independent of short-distance contributions, which are model dependent, and so provide us with a powerful tool that can be used to verify whether any model is consistent with the chiral symmetry properties of QCD.

\section{Phenomenology of meson-baryon splitting functions}

In this section we explore the features of the mesonbaryon splitting functions for the various octet and decuplet contributions that are nonzero at $y>0$, for a finite dipole cutoff parameter $\Lambda$ in Eq. (56). For illustration, we consider the nucleon and lightest $\Lambda$ hyperon states for the octet baryons, and the $\Delta$ and $\Sigma^{*}$ for the decuplet states. Unless otherwise indicated, we will use a typical value for the cutoff mass of $\Lambda=1 \mathrm{GeV}$.

In Fig. 2 we show the basis splitting functions for the onshell $f_{B, T}^{\text {(on })}$, off-shell $f_{B, T}^{\text {(off) }}$, and nonlocal off-shell $\delta f_{B, T}^{\text {(off) }}$ contributions, as well as the on-shell and off-shell endpoint functions $f_{T}^{\text {(on end) }}$ and $f_{T}^{\text {(off end) }}$ for the decuplet $\Delta$ and $\Sigma^{*}$ states. For all baryon intermediate states, the on-shell functions $f_{B, T}^{(\text {on })}$ are positive at all $y$ values and peak at around $y=0.1-0.2$, depending on the mass of the baryon. The main difference between the on-shell functions for the different baryons is the magnitude: for the strange baryons the functions are approximately an order of magnitude smaller than for the nonstrange.

The off-shell functions $f_{B, T}^{\text {(off) }}$ for the octet baryons are negative, with magnitude comparable to the on-shell functions. For decuplet baryons, the off-shell functions increase as $y \rightarrow 0$, and in fact dominate the small- $y$ region. The nonlocal off-shell functions $f_{B, T}^{\text {(off) }}$ have the same sign as the on-shell contributions, but are somewhat smaller in magnitude. The additional on-shell and off-shell endpoint contributions $f_{T}^{\text {(on end) }}$ and $f_{T}^{\text {(off end) }}$ for the decuplet intermediate states in Eqs. (88) and (104) are positive and negative, respectively, with the former vanishing at $y=0$ and the latter increasing in magnitude as $y \rightarrow 0$.

Interestingly, both the on-shell and off-shell endpoint functions at $\Lambda=1 \mathrm{GeV}$ peak at rather small values of $y$, while formally they become $\delta$-functions at $y=1$ for $\Lambda \rightarrow \infty$. The dramatic change in the shape of the endpoint functions with increasing $\Lambda$ is illustrated in Fig. 3, which shows the on-shell and off-shell endpoint terms as a function of $y$ for a range of $\Lambda$ values from $1 \mathrm{GeV}$ to $1 \mathrm{TeV}$. Of course, in practical calculations relevant for phenomenological applications, the relevant values of $\Lambda$ would typically be of the order of hadronic scales, $\sim 1 \mathrm{GeV}$; the results for the larger $\Lambda$ values shown in Fig. 3 are simply to track numerically the evolution of the nonlocal results to the local limit. 
(a)

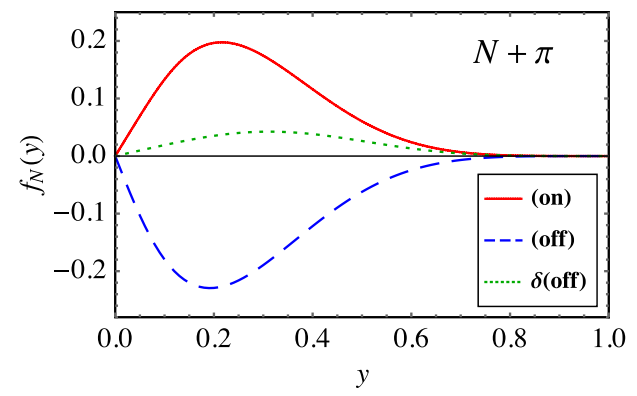

(c)

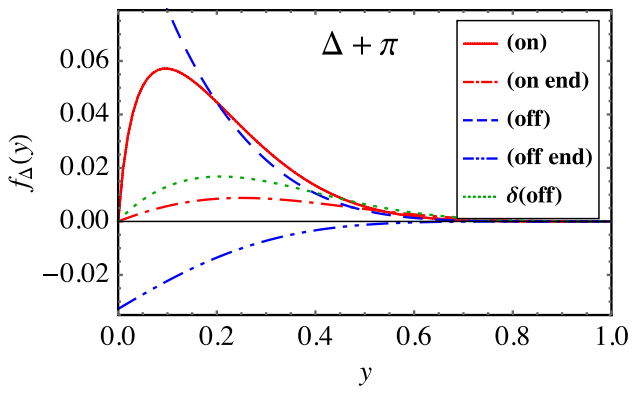

(b)

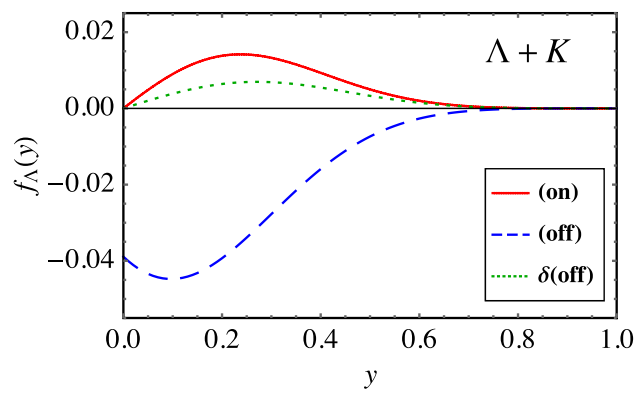

(d)

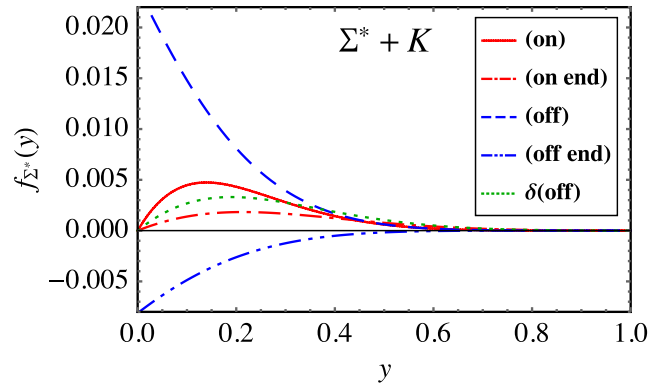

FIG. 2. Splitting functions versus meson momentum fraction $y$ for the proton dissociations into (a) $N+\pi$, (b) $\Lambda+K$, (c) $\Delta+\pi$, and (d) $\Sigma^{*}+K$ state, for the on-shell $f^{\text {(on) }}$ (red solid curves), off-shell $f^{\text {(off) }}$ (blue dashed), and nonlocal off-shell $\delta f^{\text {(off) }}$ (black dotted) contributions. For the decuplet $\Delta$ and $\Sigma^{*}$ states, additional contributions from on-shell endpoint $f^{\text {(on end) }}$ (red dot-dashed) and off-shell

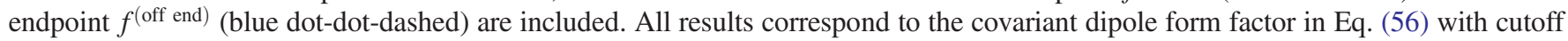
mass $\Lambda=1 \mathrm{GeV}$.

Note that the derivation of the local limit of the endpoint splitting functions, as in Eq. (89), includes the $D_{0}$ term. There, it was assumed that the constant $\Omega_{0}$ in $D_{0}$ is very large, although in the local limit it also satisfies $\Omega_{0} \ll \Lambda^{2}$ [see Eq. (91)]. In order to observe the $D_{0}$ contribution to Eq. (90) in practice, we fix the parameter $\Omega_{0}$ to a very large value, $\Omega_{0}=100 \mathrm{GeV}^{2}$. As shown in Fig. 3, when $\Lambda$ is small, the contribution of $D_{0}$ is negligible, and the on-shell and off-shell endpoint distributions coincide with those in Fig. 2(c) for $\Lambda=1 \mathrm{GeV}$. (The endpoint functions decrease in magnitude at $y<1$ with increasing $\Lambda$, so for clarity these are normalized by their integrals, $\left\langle f_{i}\right\rangle$, over all $y$. This then renders the ratio for the off-shell end point function in
Fig. 3(b) positive, whereas the unnormalized distribution in Fig. 2(c) is negative.) The $D_{0}$ term can therefore be dropped when considering the contribution of the nonlocal endpoint functions for finite values of $\Lambda$. On the other hand, Fig. 3 clearly indicates that as $\Lambda \rightarrow \infty$ the peaks of the endpoint functions migrate to higher values of $y$, approaching a shape that resembles a $\delta$-function, $\delta(1-y)$, in the local limit.

The combinations of the various basis functions corresponding to the rainbow and KR diagrams in Fig. 1 are illustrated in Fig. 4 for the same intermediate states as in Fig. 2. Again the main difference between the nonstrange and strange baryon contributions is the magnitude of the (a)

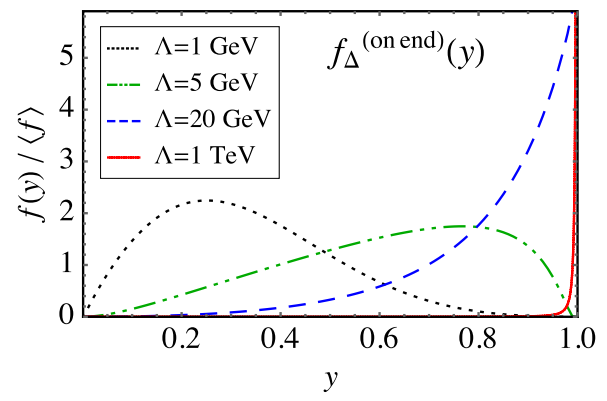

(b)

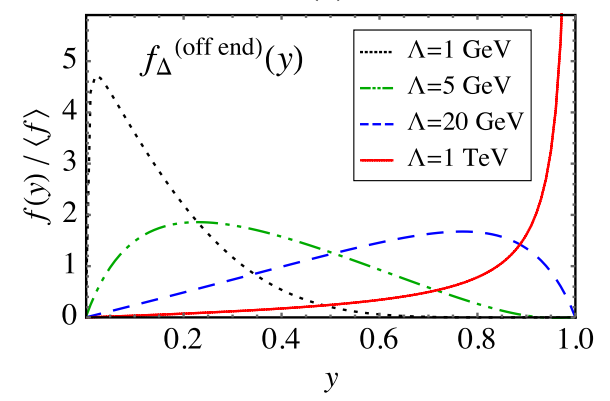

FIG. 3. Normalized splitting functions $f_{i}(y) /\left\langle f_{i}\right\rangle$ for the (a) on-shell endpoint and (b) off-shell endpoint contributions for the $\Delta+\pi$ intermediate state, for different values of the dipole cutoff mass $\Lambda(1 \mathrm{GeV}$ to $1 \mathrm{TeV})$ and a fixed value of the constant $\Omega_{0}=100 \mathrm{GeV}^{2}$. 
(a)

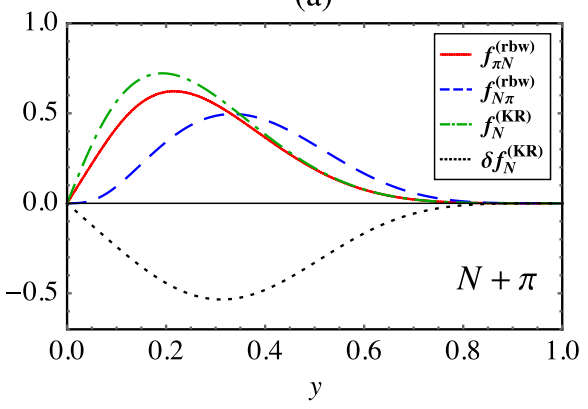

(c)

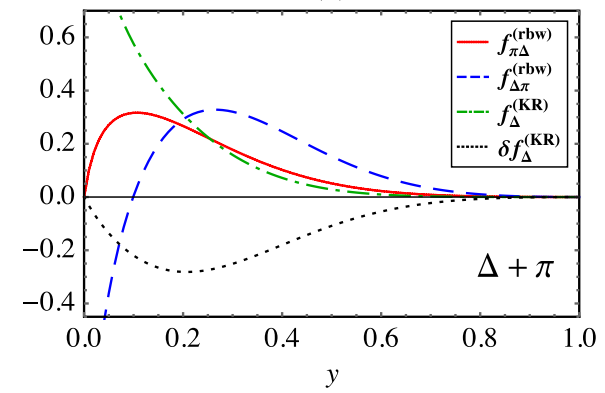

(b)

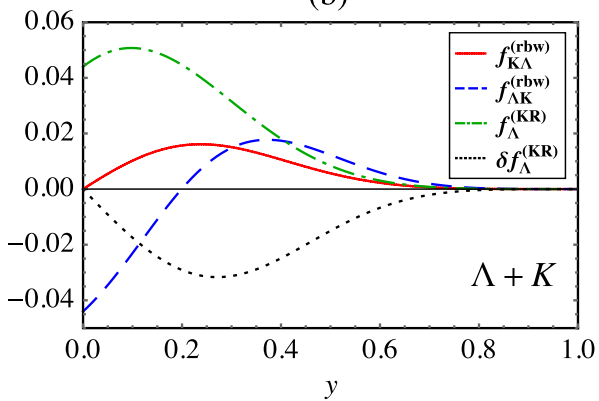

(d)

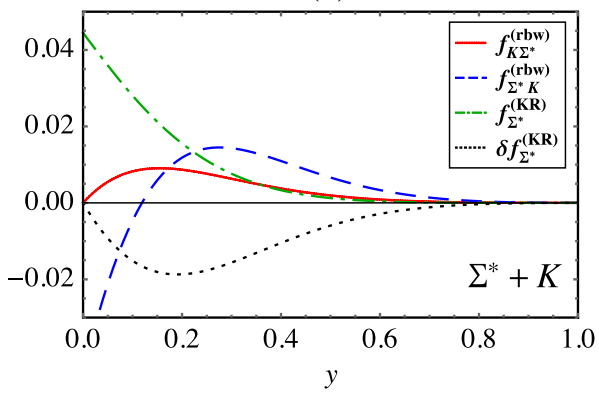

FIG. 4. Splitting functions versus $y$ for proton dissociations into various meson-baryon intermediate states as in Fig. 2, but for the total contributions to the meson-coupling rainbow diagrams in Fig. 1(a) and (h) (red solid curves), baryon-coupling rainbow diagrams in Fig. 1(b) and (i) (blue dashed), KR diagrams in Fig. 1(c) and (j) (green dot-dashed), and nonlocal KR diagrams in Fig. 1(d) and (k) (black dotted). Contributions from the tadpole and bubble diagrams in Fig. 1(e)-(g) at $y=0$ are not shown here.

functions, with the strange being an order of magnitude or more suppressed. The total meson-coupling rainbow functions, $f_{\phi B}^{(\mathrm{rbw})}$ and $f_{\phi T}^{(\mathrm{rbw})}$, generally have very similar shape to the corresponding on-shell functions in Fig. 2. The baryoncoupling rainbow functions, $f_{B \phi}^{(\mathrm{rbw})}$ and $f_{T \phi}^{(\mathrm{rbw})}$, have similar magnitude and are generally positive at intermediate $y$, but become more negative as $y \rightarrow 0$. The latter behavior is canceled by the KR functions $f_{B, T}^{(\mathrm{KR})}$ at small $y$, especially for the decuplet contributions, such that the sum of the baryon-coupling rainbow and KR diagrams satisfies Eqs. (78) and (112). The nonlocal KR functions, $\delta f_{B, T}^{(\mathrm{KR})}$, at nonzero $y$ values are proportional to -4 times the nonlocal off-shell functions [Eqs. (77) and (111)], and hence are negative at $y>0$. Some degree of cancelation therefore takes place between the local $f_{B, T}^{(\mathrm{KR})}$ and nonlocal $\delta f_{B, T}^{(\mathrm{KR})}$ functions at intermediate and large values of $y$.

The pattern of cancelations between the various contributions from the basis functions to particular diagrams in Fig. 1 is further explored in Fig. 5, which shows the decomposition of the splitting function for the nucleoncoupling rainbow diagram, $f_{N \pi}^{(\mathrm{rbw})}$. For the case of the covariant dipole form factor with $\Lambda=1 \mathrm{GeV}$, Fig. 5(a), one observes very strong cancelation between the positive (a)

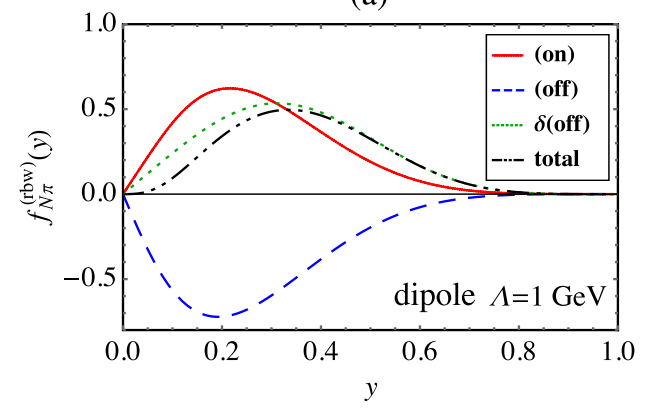

(b)

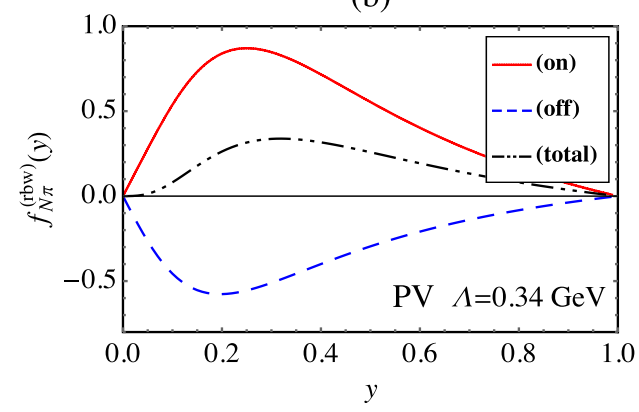

FIG. 5. Decomposition of the splitting function for the nucleon-coupling rainbow diagram in Fig. 1(b) for (a) the nonlocal chiral theory with dipole regulator, and (b) the local chiral theory with a symmetry preserving Pauli-Villars regulator. The value of the Pauli-Villars mass parameters $\Lambda$ is determined by normalizing to the momentum carried by the interacting nucleon, $\langle y\rangle=\int_{0}^{1} d y y f(y)$, for the dipole regulator with $\Lambda=1 \mathrm{GeV}$. 
(a)

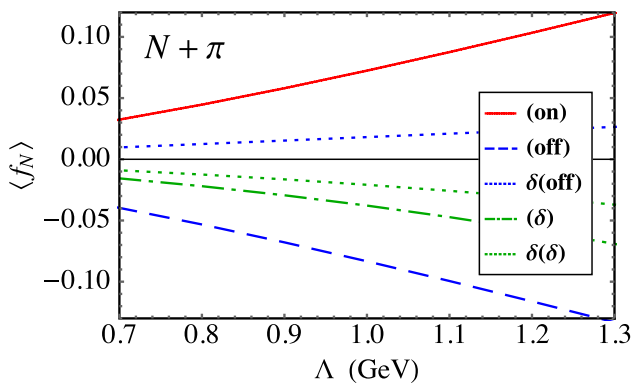

(b)

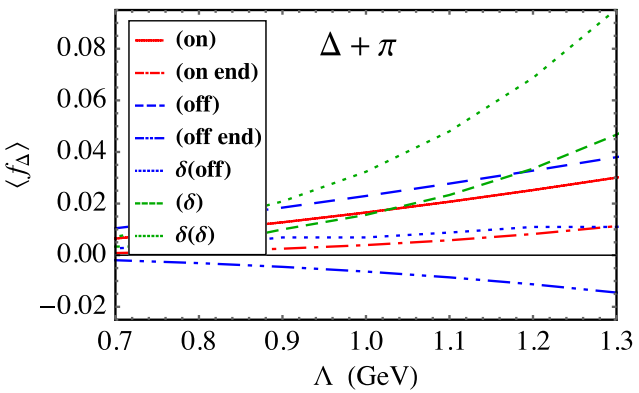

(c)

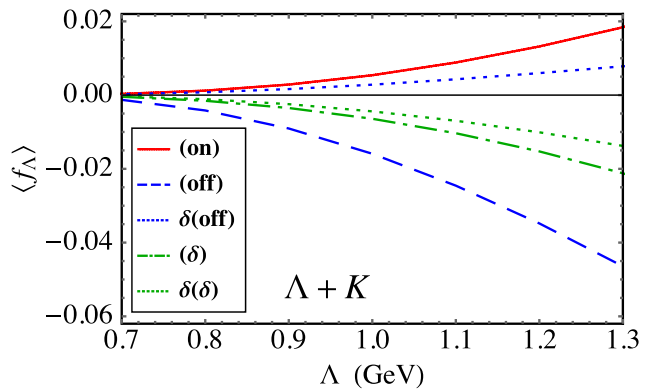

(d)

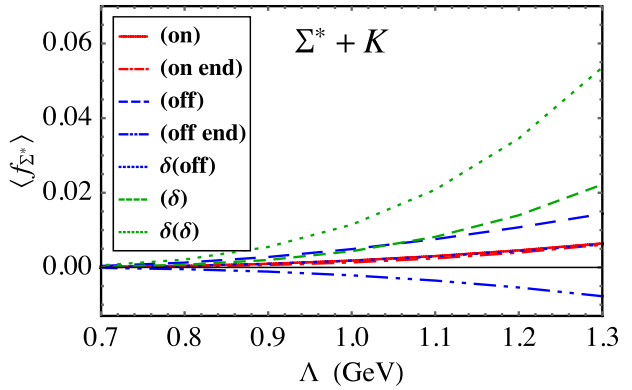

FIG. 6. Integrals of splitting functions $\langle f\rangle$ versus $\Lambda$, for (a) $N+\pi$, (b) $\Lambda+K$, (c) $\Delta+\pi$ and (d) $\Sigma^{*}+K$ intermediates states, for the on-shell (red solid curves), off-shell (blue dashed), nonlocal off-shell (blue dotted), local $\delta$-function (green dot-dashed), and nonlocal $\delta$ function (green dotted) contributions. The decuplet states include additional contributions from on-shell endpoint (red dot-dot-dashed) and off-shell end point (blue dot-dot-dashed) terms. All results correspond to the covariant dipole form factor in Eq. (56).

on-shell and negative off-shell contributions, with the total closely resembling the purely nonlocal off-shell function $\delta f^{\text {(off) }}$. At first sight this may be perplexing, if one interprets the result to suggest that the total nucleoncoupling rainbow function may be very small in the pointlike limit, where $\delta f^{\text {(off) }}$ vanishes. In practice, however, the on-shell and off-shell functions vary differently with $\Lambda$, so that the degree of cancelation depends on the cutoff.

This is illustrated in Fig. 5(b), which shows the decomposition of $f_{N \pi}^{(\mathrm{rbw})}$ for the case of a local theory with a PauliVillars regulator, which preserves the necessary symmetries of the theory $[28,29]$. In this case there is no nonlocal contribution, and the total is given by the sum of the onshell and off-shell terms. For the on-shell splitting function $f_{N}^{(\text {on) }}$ the Pauli-Villars regulating function takes the form

$$
\widetilde{F}_{\mathrm{PV}}^{\text {(on) }}(k)=1-\frac{D_{\phi B}^{2}}{D_{\Lambda_{\mathrm{PV}}}^{2}},
$$

while for the off-shell splitting function $f_{N}^{\text {(off) }}$ the regulator is given by

$$
\widetilde{F}_{\mathrm{PV}}^{(\text {off })}(k)=1-\frac{D_{\phi B}}{D_{\Lambda_{\mathrm{PV}}}}
$$

In order to compare the shapes more directly, we choose the Pauli-Villars regulator to give the same total momentum $\langle y\rangle=\int_{0}^{1} d y y f(y)$ carried by the interacting nucleon in

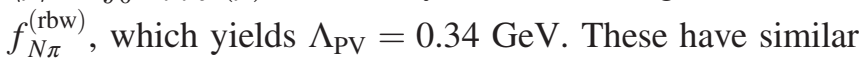
general features as the functions for the nonlocal theory with covariant dipole regulator, with the small differences in magnitude for the on-shell and off-shell contributions for the dipole and Pauli-Villars regulators allowing a sizeable nonzero total to remain.

While the contributions of the various splitting functions at $y>0$ are illustrated in Figs. 2 and 4, the relative importance of the $\delta$-functions terms at $y=0$ is demonstrated in Fig. 6 by the integrated values of the basis functions, $\langle f\rangle$ as a function of the covariant dipole form factor cutoff mass $\Lambda$. As expected, the magnitude of each of the integrated functions increases with $\Lambda$, as more shortdistance contributions are included. For the nominal $\Lambda=$ $1 \mathrm{GeV}$ used in Figs. 2 and 4 the $\pi N$ intermediate states dominate, with the hyperon and decuplet contributions an order of magnitude smaller. The picture changes for larger cutoff values, and for $\Lambda \gtrsim 1.2 \mathrm{GeV}$ some of the $\pi \Delta$ contributions become as large as the $\pi N$. Of course, the validity of a one-loop calculation for larger cutoffs is more questionable, as contributions from higher-order terms become increasingly more important. Interestingly, for the octet baryons, the on-shell and nonlocal off-shell contributions are positive, while the local off-shell and both the (local and nonlocal) $\delta$-function contributions are negative. In contrast, for the decuplet states, all contributions are positive, with the exception of the off-shell end point terms, as already indicated in Fig. 2. 


\section{CONCLUSION}

In this paper we have for the first time used a nonlocal covariant formulation of $\mathrm{SU}(3)$ chiral effective theory to construct the framework necessary for systematically computing the contributions from pseudoscalar meson loops to parton distributions in the nucleon. The main result of the present work has been the derivation from the nonlocal theory of the lowest order proton $\rightarrow$ meson + baryon splitting functions arising from transitions of the initial state to intermediate states involving octet and decuplet baryons, as well as those involving contact interactions at zero momentum.

Since the contributions from the loop diagrams are ultraviolet divergent, care must be taken to ensure that the integrals are regularized in a way that preserves the underlying symmetries of the effective theory, such as gauge invariance, Lorentz invariance, and chiral symmetry. A common approach adopted in the literature involves the use of local interactions with regulators that explicitly depend on the 3-momentum of the meson. While this does take into account the extended nature of hadrons and renders finite results, this approach is in practice $a d h o c$ and destroys the local gauge and Lorentz invariance of the theory.

The virtue of the nonlocal formulation, on the other hand, is that it allows the use of a 4-dimensional regulator while preserving the gauge and Lorentz symmetries. In this case the regulator is generated directly from the nonlocal Lagrangian, and gives rise to additional diagrams that appear from the expansion of the gauge link [see Fig. 1(d), (g) and (k)].

To illustrate the characteristic features of the new nonlocal splitting functions, we have used a simple dipole function for the 4-dimensional regulator. The approach is analogous to a resummation of chiral perturbation theory using dimensional regularization, which is known to provide better convergence at larger momenta, at the expense of losing the power counting of the traditional chiral perturbation theory. Our results reveal some novel patters of cancelations among the local and nonlocal functions in the rainbow and Kroll-Ruderman diagrams, and illustrate the importance of nonlocal contributions for finite values of the regulator mass $\Lambda$. For the decuplet intermediate states, our analysis is able to study numerically the transition from the case of a finite $\Lambda$ to the pointlike limit, which is realized most dramatically for the on-shell and off-shell end point contributions to the baryoncoupling rainbow and Kroll-Ruderman diagrams. We verify explicitly that in the $\Lambda \rightarrow \infty$ limit the nonlocal generalization does indeed reproduce the results of the local theory.

The results derived here will serve as a basis for future applications of the formalism to computing meson loop contributions to parton distributions in the nucleon. Within the effective theory, these can be computed by matching twist-two quark level and effective hadronic level operators, which leads to a convolution representation for the PDFs,

$$
q(x)=\sum_{j} \int_{0}^{1} \frac{d y}{y} f_{j}(y) q_{j}^{v}\left(\frac{x}{y}\right),
$$

where $f_{j}(y)$ are the meson-baryon splitting functions, and $q_{j}^{v}$ is the valence distribution for the quark flavor $q$ in the hadronic configuration $j$. In a forthcoming paper [43], we will use this formalism to study flavor asymmetries in the nucleon generated through meson loops, such as in the light antiquark sea $(\bar{d}-\bar{u})$ or for strange quarks $(s-\bar{s})$, consistently within the 4-dimensional chiral effective theory framework.

\section{ACKNOWLEDGMENTS}

We thank Xuangong Wang for helpful discussions. This work is supported by NSFC under Grant No. 11475186, the Sino-German CRC 110 "Symmetries and the Emergence of Structure in QCD" project by NSFC under the Grant No. 11621131001, the DOE Contract No. DE-AC0506OR23177, under which Jefferson Science Associates, LLC operates Jefferson Lab; DOE Contract No. DEFG02-03ER41260; the Australian Research Council through the ARC Centre of Excellence for Particle Physics at the Terascale (CE110001104); and an ARC Discovery Project No. DP151103101.
[1] J. Speth and A. W. Thomas, Adv. Nucl. Phys. 24, 83 (1997).

[2] S. Kumano, Phys. Rep. 303, 183 (1998).

[3] A. W. Thomas, Phys. Lett. 126B, 97 (1983).

[4] A. Baldit et al., Phys. Lett. B 332, 244 (1994).

[5] R. S. Towell et al., Phys. Rev. D 64, 052002 (2001).

[6] M. Arneodo et al., Phys. Rev. D 50, R1 (1994).

[7] K. Ackerstaff et al., Phys. Rev. Lett. 81, 5519 (1998).
[8] A. I. Signal and A. W. Thomas, Phys. Lett. B 191, 205 (1987),

[9] D. Mason et al., Phys. Rev. Lett. 99, 192001 (2007).

[10] S. Alekhin, S. A. Kulagin, and R. Petti, Phys. Lett. B 675, 433 (2009).

[11] J. J. Ethier, N. Sato, and W. Melnitchouk, Phys. Rev. Lett. 119, 132001 (2017).

[12] A. W. Schreiber, A. I. Signal, and A. W. Thomas, Phys. Rev. D 44, 2653 (1991). 
[13] W. Melnitchouk and A. W. Thomas, Z. Phys. A 353, 311 (1995).

[14] F. M. Steffens, H. Holtmann, and A. W. Thomas, Phys. Lett. B 358, 139 (1995).

[15] D. Diakonov, V. Petrov, P. Pobylitsa, M. V. Polyakov, and C. Weiss, Nucl. Phys. B480, 341 (1996).

[16] W. Melnitchouk and M. Malheiro, Phys. Rev. C 55, 431 (1997).

[17] W. Melnitchouk and M. Malheiro, Phys. Lett. B 451, 224 (1999).

[18] F. Myhrer and A. W. Thomas, Phys. Lett. B 663, 302 (2008).

[19] A. W. Thomas, W. Melnitchouk, and F. M. Steffens, Phys. Rev. Lett. 85, 2892 (2000).

[20] D. Arndt and M. J. Savage, Nucl. Phys. A697, 429 (2002).

[21] J. W. Chen and X. Ji, Phys. Lett. B 523, 107 (2001).

[22] J.-W. Chen and X. Ji, Phys. Rev. Lett. 87, 152002 (2001); 88, 249901(E) (2002).

[23] W. Detmold, W. Melnitchouk, J. W. Negele, D. B. Renner, and A. W. Thomas, Phys. Rev. Lett. 87, 172001 (2001).

[24] M. Burkardt, K. S. Hendricks, C.-R. Ji, W. Melnitchouk, and A. W. Thomas, Phys. Rev. D 87, 056009 (2013).

[25] C.-R. Ji, W. Melnitchouk, and A. W. Thomas, Phys. Rev. D 88, 076005 (2013).

[26] A. M. Moiseeva and A. A. Vladimirov, Eur. Phys. J. A 49, 23 (2013).

[27] Y. Salamu, C.-R. Ji, W. Melnitchouk, and P. Wang, Phys. Rev. Lett. 114, 122001 (2015).

[28] X. G. Wang, C.-R. Ji, W. Melnitchouk, Y. Salamu, A. W. Thomas, and P. Wang, Phys. Lett. B 762, 52 (2016).

[29] X. G. Wang, C.-R. Ji, W. Melnitchouk, Y. Salamu, A. W. Thomas, and P. Wang, Phys. Rev. D 94, 094035 (2016).

[30] A. W. Thomas, Nucl. Phys. B, Proc. Suppl. 119, 50 (2003).

[31] A. W. Thomas, Adv. Nucl. Phys. 13, 1 (1984).

[32] J. Terning, Phys. Rev. D 44, 887 (1991).

[33] K. Wilson, Phys. Rev. D 10, 2445 (1974).

[34] C. Bloch, K. Dan. Vidensk. Selsk. Mat. Fys. Medd. 26, 1 (1950).

[35] H. Forkel, M. Nielsen, X.-M. Jin, and T. D. Cohen, Phys. Rev. C 50, 3108 (1994).
[36] M. J. Musolf and M. Burkardt, Z. Phys. C 61, 433 (1994).

[37] S. Wang and M. K. Banerjee, Phys. Rev. C 54, 2883 (1996).

[38] B. Holdom, Phys. Rev. D 45, 2534 (1992).

[39] A. Faessler, T. Gutsche, M. A. Ivanov, V. E. Lyubovitskij, and P. Wang, Phys. Rev. D 68, 014011 (2003).

[40] P. Wang, Eur. Phys. J. A 50, 172 (2014).

[41] F. C. He and P. Wang, Phys. Rev. D 97, 036007 (2018).

[42] F. C. He and P. Wang, Phys. Rev. D 98, 036007 (2018).

[43] Y. Salamu, C.-R. Ji, W. Melnitchouk, A. W. Thomas, P. Wang, and X. Wang (to be published).

[44] E. E. Jenkins, Nucl. Phys. B368, 190 (1992).

[45] T. Ledwig, J. Martin Camalich, L. S. Geng, and M. J. Vicente Vacas, Phys. Rev. D 90, 054502 (2014).

[46] T. R. Hemmert, B. Kubis, and U.-G. Meissner, Phys. Rev. C 60, 045501 (1999).

[47] T. R. Hemmert, U.-G. Meissner, and S. Steininger, Phys. Lett. B 437, 184 (1998).

[48] A. V. Manohar, Lect. Notes Phys. 479, 311 (1997).

[49] R. D. Young, D. B. Leinweber, and A. W. Thomas, Prog. Part. Nucl. Phys. 50, 399 (2003).

[50] J. M. M. Hall, D. B. Leinweber, and R. D. Young, Phys. Rev. D 82, 034010 (2010).

[51] R. Tegen and W. Weise, Z. Phys. A 314, 357 (1983).

[52] C. Hacker, N. Wies, J. Gegelia, and S. Scherer, Phys. Rev. C 72, 055203 (2005).

[53] L. M. Nath, B. Etemadi, and J. D. Kimel, Phys. Rev. D 3, 2153 (1971).

[54] W. Melnitchouk, A. W. Thomas, and A. I. Signal, Z. Phys. A 340, 85 (1991).

[55] M. Burkardt and Y. Koike, Nucl. Phys. B632, 311 (2002).

[56] H. Holtmann, A. Szczurek, and J. Speth, Nucl. Phys. A596, 631 (1996).

[57] W. Melnitchouk, J. Speth, and A. W. Thomas, Phys. Rev. D 59, 014033 (1998).

[58] X. Wang (private communication).

Correction: A second affiliation has been inserted for the first author. 